Article

\title{
Time-Sharing Control Strategy for Multiple-Receiver Wireless Power Transfer Systems
}

\author{
Weikun Cai $\odot$, Dianguang Ma *, Xiaoyang Lai ${ }^{*}$, Khurram Hashmi $\odot$, Houjun Tang and \\ Junzhong Xu
}

Key Laboratory of Control of Power Transmission and Transformation Ministry of Education, Shanghai Jiao Tong University, 800 Dongchuan RD., Shanghai 200240, China; yezide@sjtu.edu.cn (W.C.); khurram_hashmi@sjtu.edu.cn (K.H.); hjtang@sjtu.edu.cn (H.T.); junzhongxu@sjtu.edu.cn (J.X.)

* Correspondence: dgma@sjtu.edu.cn (D.M.); laixy@sjtu.edu.cn (X.L.); Tel.: +86-13817587820 (D.M.); +86-13918001061 (X.L.)

Received: 31 December 2019; Accepted: 24 January 2020; Published: 29 January 2020

\begin{abstract}
The cross-coupling effect between the induction coils of a multiple-receiver wireless power transfer (MRWPT) system severely weakens its overall performance. In this paper, a time-sharing control strategy for MRWPT systems is proposed to reduce the cross-coupling between receiver coils. An active-bridge rectifier is introduced to the receivers to replace the uncontrollable rectifier to achieve synchronization of the time-sharing control. The synchronization signal generated by an active-bridge rectifier can be directly used to realize the synchronization of time-sharing control and hence saved the traditional zero-crossing point detection circuits for time-sharing circuits. Moreover, the proposed time-sharing system has the advantages of both operating under a resistance-matching condition and providing target output voltage for each receiver. Furthermore, a voltage control strategy was developed to provide both high efficiency and a target output voltage for each receiver. Finally, the simulation and experimental results show that the time-sharing MRWPT system reduced the cross-coupling effect between the receiver coils, and the voltage control strategy provided both a high efficiency and a target output voltage for each receiver.
\end{abstract}

Keywords: wireless power transfer; multiple receiver; cross-coupling

\section{Introduction}

Wireless power transfer (WPT) is a promising technology that eliminates the need for transmission cables leading to electrical devices and makes power transfer more convenient. WPT technology has recently attracted increased attention in industry and academia where it has been widely applied to areas like underwater systems [1,2], biomedical implants [3-6], mining devices [7,8], smart robots [9], and electronic equipment [7]. With the increasing demands for embedding multiple electrical devices in a WPT system, the technologies in a multiple-receiver wireless power transfer (MRWPT) system are becoming advanced. Multiple types of equipment embedded into a WPT system mostly operates with different loading characters and different output voltages. Further research may be conducted to simultaneously achieve both high efficiency and target output voltages. Furthermore, the cross-coupling between the receiver coils severely weakens the system's efficiency and decreases the output voltages when the receiver coils are in proximity. More research is needed to improve the compensation for the cross-coupling effect between receivers.

Some studies have focused on improving the system efficiency and providing voltage control on MRWPT systems, as discussed in References [10-15]. A MRWPT system [10] is proposed for portable devices with simplified receiver design. In Cannon et al. [11], multiple receiver systems with resonant coupling are constructed with a low complexity circuit and a resonant frequency splitting effect is 
observed in MRWPT systems. To increase the system's efficiency, impedance matching theory [12] is analyzed within an MRWPT system. Moreover, metamaterials are introduced to the MRWPT system to improve the system's efficiency, and as a result, the efficiency is improved by approximately $20 \%$ [13]. To provide a high efficiency and a transfer target output power in a two-receiver WPT system, a control method based on a simplified math model was developed to meet the demands of different load characteristic [14]. To meet the constant output voltage commands, a load-independent voltage method was analyzed in Sun et al. [15].

WPT systems achieve maximum power transfer efficiency when the load resistances comply with the impedance matching methods [12]. To increase the system's efficiency, multiple topologies and control strategies have been proposed for WPT systems. The optimal impedances for the MRWPT systems have been investigated and buck circuits are employed in receivers to manipulate the equivalent load resistances [16]. The system achieves an efficiency of $80 \%$ at the frequency $13.56 \mathrm{MHz}$. However, the output voltage cannot be controlled because the buck circuit must meet the optimal load demand. To manipulate the load resistance for the impedance matching method, a buck-boost topology [17] is introduced to the rectifier for optimizing the load resistances. The system achieves maximum efficiency of $78 \%$ when transferring $16 \mathrm{~W}$ of power. An amplitude control strategy with a phase-shifting method [18] is introduced to control the equivalent load resistance for impedance matching. The maximum efficiency is about $36 \%$ with a factor $k=0.6$ and can reach $83 \%$ with a factor $k=0.3$. A buck converter [19] is introduced to the transmitter to regulate the input power and a boost converter is constructed at the receiver to manipulate the load resistance. The system transfers $100 \mathrm{~W}$ with an efficiency of about $79 \%$. Dual-active bridge circuits $[20,21]$ are introduced to the WPT systems to displace the uncontrolled rectifier. Compared to the uncontrolled rectifier, a dual-active bridge has a smaller conduction resistance and can manipulate the load resistance. The system achieved an improved efficiency of $85.4 \%$ in Li et al. [20], while Liu et al. [21] achieved an efficiency of $80.4 \%$. However, the performances of these circuits are weakened by cross-coupling between coils; when these circuits are applied to MRWPT systems, the circuits should be improved to reduce the cross-coupling effect.

The cross-coupling effect between multiple receivers in MRWPT systems is surveyed in Ahn and Hong [22]. This study indicates that the resonant frequency varies for the cross-coupling effect and the compensation networks need to be readjusted correspondingly. To reduce and eliminate the cross-coupling effect, various approaches are proposed. Coupling effects are considered during an impedance matching procedure in Kim et al. [23] to improve the system efficiency. The design of the source coil and optimal load impedance are achieved based on circuit analysis. Some studies have reduced the cross-coupling effect by compensating the cross-coupling with resonance capacitors. The cross-coupling effect in the two-receiver WPT systems are analyzed and compensated to achieve a higher system efficiency [24]. Furthermore, the cross-coupling effect compensations are extended to the multiple-receiver WPT systems to reduce the cross-coupling influence on multiple receivers in Fu et al. [25]. However, the compensation methods regarding cross-coupling are based on a fixed cross-coupling value and fail to be effective for the MRWPT systems where the distances between coils are constantly changing. Further studies on cancelling the constantly changing cross-coupling effect should be investigated to improve MRWPT system efficiencies.

Other methods to cancel the cross-coupling effect are proposed for each receiver to operate on different frequencies or set each receiver to turn on in different time sequences. Due to the different resonant frequencies of each receiver, the transmitter is manipulated to transfer the specified resonant frequency to activate specified receiver in Jiang et al. [26]. Three different resonant frequencies, namely $60 \mathrm{kHz}, 100 \mathrm{kHz}$, and $140 \mathrm{kHz}$, are chosen to deliver power to three DC motors with an efficiency of about $60 \%$. Moreover, the cross-coupling effect between coils is isolated by separating the resonant frequencies in Kim et al. [27] and the separated resonant frequencies are achieved using a special compensation network. The system has an efficiency of $24 \%-29 \%$ at the resonant frequencies of $20 \mathrm{MHz}$, $22.5 \mathrm{MHz}$, and $25 \mathrm{MHz}$. A dual-frequency mode for 3-D stacked multiple receivers is developed 
in Yanagawa et al. [28] to increase the system efficiency. The dual-frequency mode is formulated using an optimization methodology involving tuning capacitor values to achieve an efficiency of $58.7 \%$ at $6.78 \mathrm{MHz}$ and $13.56 \mathrm{MHz}$. Moreover, an uncontrollable rectifier bridge is introduced to the MRWPT system to manipulate the time-division of each receiver in Yin et al. [29] and time-sharing WPT systems are developed in Jiang et al. [30] to eliminate the cross-coupling effect in DC motor drives. By introducing a time-sharing method in manipulating the compensation capacitors at the transmitter, the transmitter transfers a specific frequency in different time intervals to activate a specific receiver. Another method used to the cancel cross-coupling effect is proposed in Juliet et al. [31] by compensating cross-coupling with an additional relay coil and independent resonance frequency tuning. The system operates under $1 \mathrm{MHz}, 1.5 \mathrm{MHz}$, and $3 \mathrm{MHz}$, eliminating the frequency splitting effect and reducing the cross-coupling effect in the MRWPT systems. However, these methods are based on operating at different resonant frequency and are not applicable for single-band applications.

In this paper, the topology of a time-sharing multiple-receiver wireless power transfer (TS-MRWPT) system with an active-bridge rectifier is developed to reduce the effect of cross-coupling between receiver coils, and a synchronization strategy is developed to control the time-sharing sequence of each receiver. Moreover, a voltage control strategy is proposed to achieve both a high system efficiency and to satisfy the output voltage demands for each receiver.

The organization of this paper is as follows: Section 2 analyzes the mathematical model and cyclic control strategy of TS-MRWPT systems with uncontrollable rectifiers. Section 3 develops a topology of TS-MRWPT systems with active-bridge rectifiers, proposes a synchronization strategy for the time-sharing sequence of each receiver, and analyzes the drive signal generation and influences brought about by RC absorbing circuits. The output voltage control strategy is developed for the proposed topology to achieve both a high efficiency and satisfying the output voltage demands of each receiver. Section 4 presents the simulation and experiment results, and finally, the conclusion is given in Section 5.

\section{TS-MRWPT System with Uncontrollable Rectifiers}

\subsection{Modeling of a TS-MRWPT System with Uncontrolled Rectifiers}

Figure 1 displays the schematic and equivalent circuits of the time-sharing MRWPT system with uncontrolled rectifiers. $R_{i}, L_{i}$, and $C_{i}$ represent the resistance, inductance, and compensation capacitance of the $i$ th coil, respectively. $R_{\mathrm{o} i}$ denotes the load resistance of coil $i, C_{\mathrm{o} i}$ denotes the filter capacitance of coil $i$, and $U_{\text {in }}$ denotes the input DC voltage. $U_{\mathrm{o} i}$ denotes the DC output voltage of the load resistance $R_{\mathrm{o} i}$ in coil $i . Q_{11}-Q_{14}$ are the gate drive signals of the converters of coil 1. $D_{i}$ indicates the duty cycle of $S_{i 5}$ and $T_{\text {rec }}$ indicates the switching cycle of the $S_{i 5}$. Frequency $f_{1}$ indicates the frequency of the inverter at the primary side. Frequency $f_{2}$ indicates the frequency of $Q_{i 5}$. $N_{f}$ indicates the ratio $f_{1} / f_{2}$.

Compared to the dual-series compensation MRPWT system in Liu et al. [21], based on the Kirchhoff voltage laws (KVLs), the equivalent mathematical model of the TS-MRWPT system can be derived as:

$$
\left[\begin{array}{ccccc}
j \omega L_{\mathrm{f} 1}+\frac{1}{j \omega C_{\mathrm{fl}}} & -\frac{1}{j \omega C_{\mathrm{f} 1}} & 0 & \cdots & 0 \\
-\frac{1}{j \omega C_{\mathrm{f} 1}} & R_{1}+j \omega L_{\mathrm{s} 1}+\frac{1}{j \omega \mathrm{C}_{\mathrm{f}}}+j \omega L_{1}+\frac{1}{j \omega C_{1}} & -j \omega M_{12} & \cdots & -j \omega M_{1 n} \\
0 & -j \omega M_{12} & R_{2}+j \omega L_{2}+\frac{1}{j \omega \mathrm{C}_{2}}+Z_{\mathrm{eq} 2} & \cdots & j \omega M_{2 n} \\
0 & -j \omega M_{13} & j \omega M_{23} & \cdots & j \omega M_{3 n} \\
\vdots & \vdots & \vdots & \ddots & \vdots \\
0 & -j \omega M_{1 n} & j \omega M_{2 n} & \cdots & R_{n}+j \omega L_{n}+\frac{1}{j \omega C_{n}}+Z_{\text {eqn }}
\end{array}\right]\left[\begin{array}{c}
I_{1} \\
I_{p 1} \\
I_{2} \\
I_{3} \\
\vdots \\
I_{n}
\end{array}\right]=\left[\begin{array}{c}
U_{1} \\
0 \\
0 \\
0 \\
0 \\
0
\end{array}\right]
$$




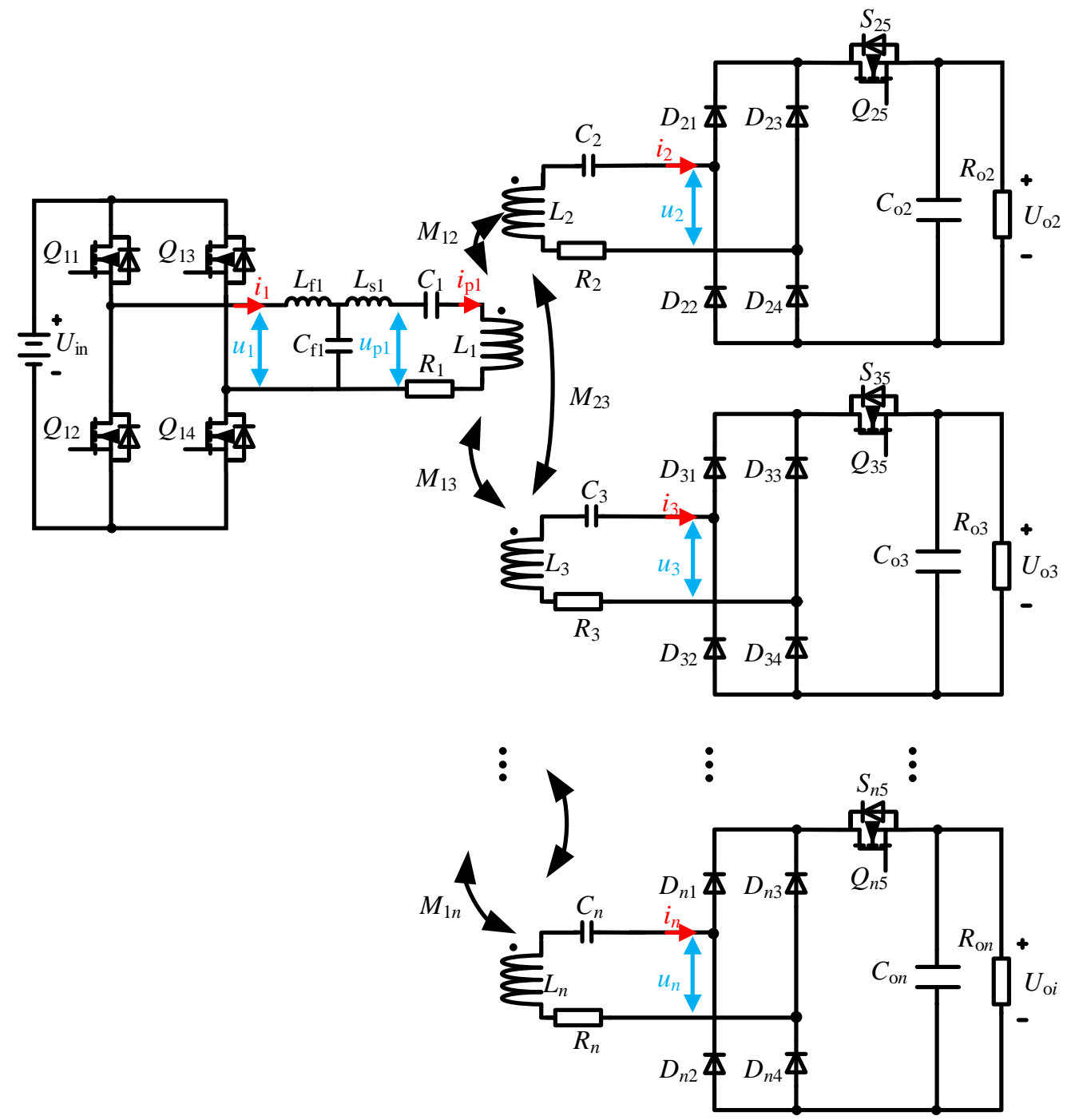

(a)
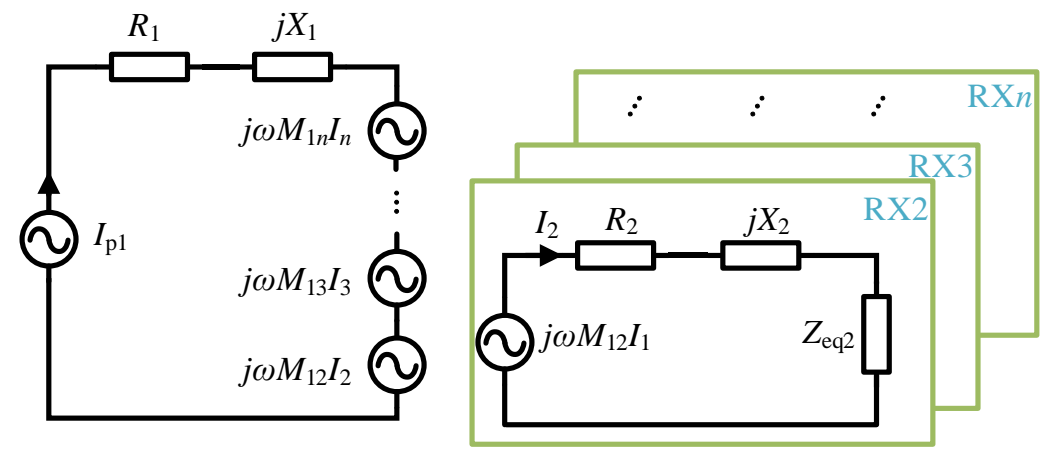

(b)

Figure 1. Schematic and equivalent circuits of a time-sharing multiple-receiver wireless power transfer (WPT) system with uncontrollable rectifiers: (a) schematic circuit and (b) equivalent circuit.

In the TS-MRWPT system, at any time, only one Metal-Oxide-Semiconductor Field-Effect Transistor (MOSFET) $S_{i 5}$ is turned on and the other $S_{i 5}(j \neq i, j=1,2, \ldots, n)$ are turned off. Therefore, the currents satisfy $I_{i} \neq 0$ and $I_{j}=0$ and Equation (1) can be simplified to (2): 


$$
\left[\begin{array}{ccc}
j \omega L_{\mathrm{f} 1}+\frac{1}{j \omega C_{\mathrm{f} 1}} & -\frac{1}{j \omega \mathrm{C}_{\mathrm{f} 1}} & 0 \\
-\frac{1}{j \omega C_{\mathrm{f} 1}} & R_{1}+j \omega L_{\mathrm{s} 1}+\frac{1}{j \omega C_{\mathrm{f} 1}}+j \omega L_{1}+\frac{1}{j \omega C_{1}} & -j \omega M_{1 i} \\
0 & -j \omega M_{1 i} & R_{i}+j \omega L_{i}+\frac{1}{j \omega C_{i}}+Z_{\mathrm{eq} i}
\end{array}\right]\left[\begin{array}{c}
I_{1} \\
I_{\mathrm{p} 1} \\
I_{i}
\end{array}\right]=\left[\begin{array}{c}
U_{1} \\
0 \\
0
\end{array}\right]
$$

In order to turn off the currents at the receiver side, the compensation network at the receiver side has to have voltage-source characteristics instead of current-source characteristics. The compensation network of the receiver side are in a series compensation circuit instead of a parallel compensation circuit or LCC compensation circuit. The compensation networks satisfy Equation (3):

$$
\omega L_{i}=\frac{1}{\omega C_{i}},(i=1,2, \cdots, n)
$$

According to the characteristics of an LCL circuit, the $L_{\mathrm{f} 1}, L_{\mathrm{s} 1}$, and $C_{\mathrm{f} 1}$ satisfy Equation (4):

$$
\omega L_{\mathrm{f} 1}=\omega L_{\mathrm{s} 1}=\frac{1}{\omega C_{\mathrm{f} 1}}
$$

The ratio $\alpha$ of current $I_{\mathrm{p} 1}$ and voltage $U_{1}$ satisfies Equation (5) based on Equations (1) and (4):

$$
\alpha=\frac{I_{\mathrm{p} 1}}{U_{1}}=\frac{1}{j \omega L_{\mathrm{f} 1}}
$$

When the filter capacitors $C_{\mathrm{o} i}(i=2,3, \ldots, n)$ are large enough, the output voltages $U_{\mathrm{o} i}$ are supposed to remain unchanged. $U_{i}(i=2,3, \ldots, n)$ indicates the fundamental component of the converter voltage at receiver side, which are derived as:

$$
U_{i}=\frac{2 \sqrt{2}}{\pi} U_{\mathrm{o} i}
$$

Supposing no extra power losses occur at the converters, the power switches $S_{i 5}$, and at the receiver side, based on the law of energy conservation, the power of the load resistances satisfies Equation (7):

$$
\int_{0}^{T_{\text {rec }}} \frac{U_{\mathrm{o} i}{ }^{2}}{R_{\mathrm{o} i}} d t=\int_{0}^{D_{i} T_{\text {rec }}} \frac{U_{i}{ }^{2}}{R_{\mathrm{eq} i}} d t
$$

By deriving Equation (7), the equivalent resistance $R_{\text {eq } i}$ of load resistance $R_{\mathrm{o} i}$ satisfies Equation (8):

$$
R_{\mathrm{eq} i}=\frac{8 D_{i}}{\pi^{2}} R_{\mathrm{o} i}
$$

Based on Equations (1) and (6), the output voltage of the $i$ th receiver coil satisfies Equation (9):

$$
U_{\mathrm{o} i}=\frac{8 M_{1 i} R_{\mathrm{o} i} U_{\mathrm{in}} \sin \beta_{1 i}}{L_{\mathrm{f} 1}\left(\pi^{2} R_{i}+8 R_{\mathrm{o} i}\right)}
$$

\subsection{Cyclic Control of a TS-MRWPT System with Uncontrollable Rectifiers}

This section proposes a cyclic control strategy for a TS-MRWPT system. This cyclic control strategy enables multiple receivers to be turned on in different time sequences, which can reduce the effect of cross-coupling between receiver coils. Meanwhile, a cyclic control strategy is a periodic control strategy that manipulates the power index of each load resistance by manipulating the amount of the full-wave of the rectifier at a switch cycle $T_{\text {rec }}$. 
Figure 2 displays the cyclic control method of a time-sharing, single-transmitter, three-receiver WPT system. The figure includes the drive signal $Q_{i 5}$ of $S_{i 5}$ and the output currents $i_{\mathrm{o} i}$ of each load resistance.

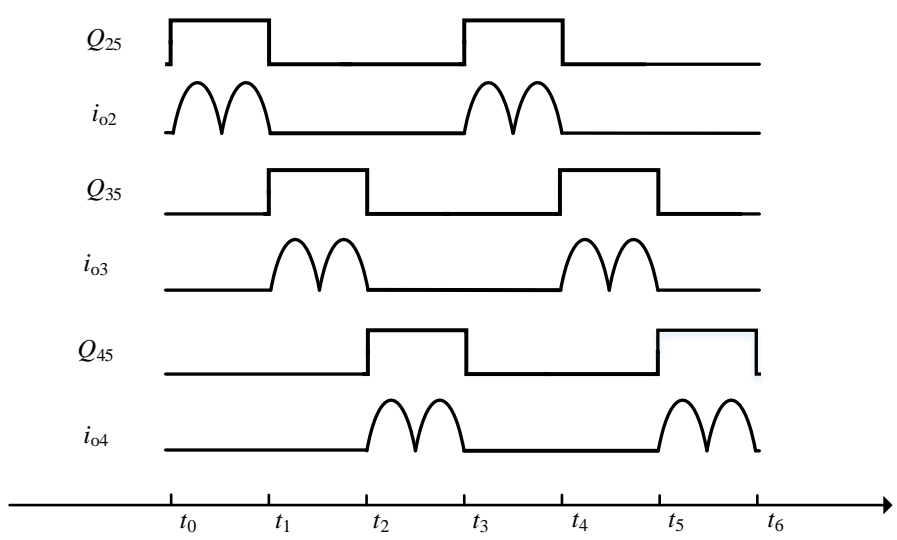

Figure 2. Cyclic control method of the single-transmitter, three-receiver, time-sharing WPT system with an uncontrollable rectifier.

There are self-inductances $L_{i}(i=2,3, \ldots, n)$ at the receiver coils. The sudden turn-off of $S_{i 5}$ will cause a large $L_{i} \frac{d i_{i}}{d t}$ at the receiver coils, which may lead to the breakdown of receiver coils. Therefore, the $S_{i 5}$ are turned off when the currents $I_{i}$ are at the zero-crossing condition.

However, the cyclic control of a TS-MRWPT system must introduce a zero-crossing current detection of $I_{i}$ to avoid a large voltage $U_{i}$ in the receiver coils. Furthermore, the tuning range of the equivalent resistances are restricted by the duty cycle $D_{i}$, and the tuning range of the output power of each load resistance $R_{\mathrm{o} i}$. New topologies and a control strategy should be proposed to improve the performance of the time-sharing MRWPT system.

\section{Time-Sharing, Multiple-Receiver, Wireless Power Transfer System with Active-Bridge Rectifiers}

To improve the performance of the TS-MRWPT system, an active-bridge rectifier is introduced to the TS-MRWPT system. An active-bridge rectifier has the advantage of lower power loss in the converters and a controllable equivalent resistance. Moreover, an active-bridge rectifier enables the MRWPT system to achieve the phases of the receiver currents $I_{i}$.

\subsection{Modeling of the TS-MRWPT with Active-Bridge Rectifiers}

Figure 3 shows the schematic of a TS-MRWPT system with active-bridge rectifiers. The uncontrollable rectifiers in Figure 1a are replaced by an active bridge in Figure 3. The active bridge is driven by drive signal $Q_{i 1}-Q_{i 5} \cdot Q_{i 1}-Q_{i 4}(i=1,2, \ldots, n)$ are the gate drive signals of the converters of the coil $i$.

Compared to the equivalent resistance $R_{\text {eqi }}$ of a TS-MRWPT system with uncontrollable rectifiers in Equation (6), the equivalent resistance $R_{\text {eqi }}$ of a TS-MRWPT system with an active bridge can be derived to give Equation (10), where $2 \beta_{i}$ denotes the conduction angle of the converters:

$$
R_{\mathrm{eq} i}=\frac{8 D_{i}}{\pi^{2}} R_{\mathrm{o} i} \sin ^{2} \beta_{i}
$$

Based on Deng et al. [32], the best matching resistances $R_{\mathrm{eqi}}$ BEST, $(i=2,3, \ldots, n)$ of an MRWPT system satisfies Equation (11):

$$
R_{\mathrm{eq} i, \mathrm{BEST}}=R_{i} \sqrt{1+\frac{1}{R_{1}}\left(\frac{\omega^{2} M_{1 i}{ }^{2}}{R_{i}}\right)}
$$



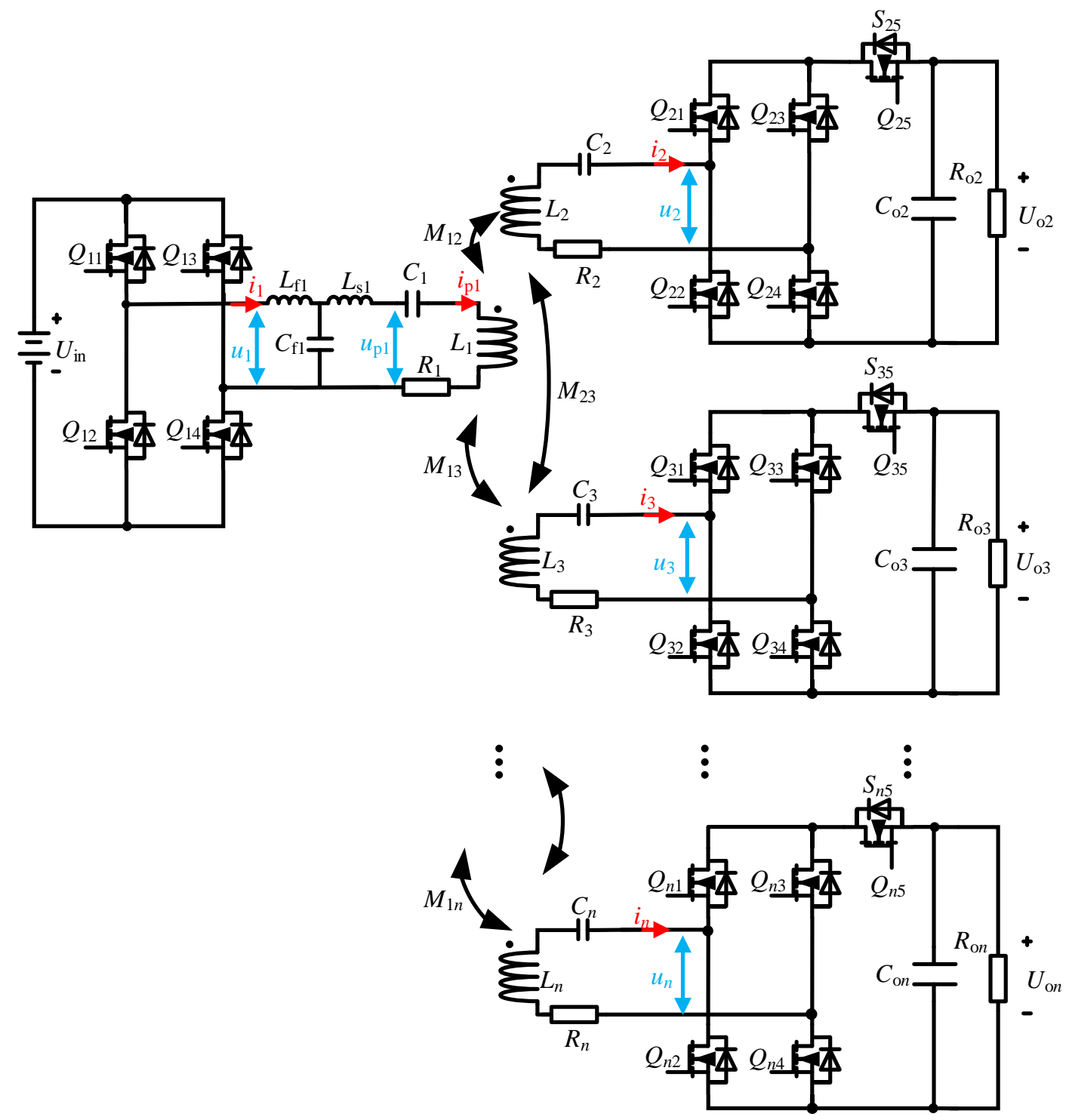

Figure 3. Schematic of a time-sharing, multiple-receiver WPT system with active-bridge rectifiers.

In Figure $3, U_{1}$ denotes the fundamental component of the inverter voltage. By performing a Fourier decomposition, $U_{i 1}$ is derived as:

$$
U_{1}=\frac{2 \sqrt{2}}{\pi} U_{\text {in }} \sin \beta_{1}
$$

Based on Liu et al. [21], the fundamental component of the converter voltage $U_{i}(i=2,3, \ldots, n)$ at the receiver is derived as:

$$
U_{i}=\frac{2 \sqrt{2}}{\pi} U_{\mathrm{o} i} \sin \beta_{i}
$$

By solving Equations (1) and (9)-(13), the output voltage of the $i$ th load resistance $U_{\mathrm{o} i}$ can be derived to give Equation (14).

$$
U_{\mathrm{o} i}=\frac{8 D_{i} M_{1 i} R_{\mathrm{o} i} U_{\mathrm{in}} \sin \beta_{1} \sin \beta_{i}}{L_{\mathrm{f} 1}\left(\pi^{2} R_{i}+8 D_{i} R_{\mathrm{o} i} \sin ^{2} \beta_{i}\right)}
$$


In practical applications, the $R_{i}$ satisfy $R_{i} \ll \frac{8 D_{i} R_{\mathrm{o} i} \sin ^{2} \beta_{i}}{\pi^{2}}$, and Equation (14) can be simplified to give Equation (15):

$$
U_{\mathrm{o} i} \approx U_{\mathrm{in}} \frac{M_{1 i} \sin \beta_{1}}{L_{\mathrm{f} 1} \sin \beta_{i}}, \text { when } R_{i} \ll \frac{8 D_{i} R_{\mathrm{o} i} \sin ^{2} \beta_{i}}{\pi^{2}}
$$

From Equation (15), the output voltage remains unchanged despite the variation of $D_{i}$. Moreover, the output voltage is controlled by $\beta_{1}$, and the equivalent resistances of the load resistances are controlled by $\beta_{1}$ and $D_{i}$ cooperatively. Therefore, the proposed topology has the advantage of a constant output voltage despite the variation of $D_{i}$ and the output voltages are consistently controlled by $\beta_{1}$ and $\beta_{i}$ $(i=2,3, \ldots, n)$. Furthermore, in practical applications, it is difficult to achieve accurate values for $D_{i}$ due to the full-wave control strategy. The full-wave control strategy must assure that the number of the turn-on waveforms of the voltage is an integer. The proposed topology can reduce the output voltage error caused by the $D_{i}$ error.

\subsection{Cyclic Control for a Time-Sharing, Multiple-Receiver, Wireless Power Transfer System with Active-Bridge Rectifiers}

This section proposes a cyclic control strategy for a TS-MRWPT system with active-bridge rectifiers. A cyclic control strategy focuses on turning off the power switches $S_{i 5}$ when $S_{i 5}$ reach their zero-crossing current conditions. The zero-crossing detection signal is generated by the synchronization signals, which are driven by the synchronization control of the active-bridge rectifiers.

This paragraph illustrates the synchronization procedure of the TS-MRWPT system. According to Liu et al. [21], the synchronization signal can be achieved by detecting the voltage $u_{C i}$. After the synchronization control of the active-bridge rectifiers, the phases of the voltage $u_{i}$ at the receiver side are the same phases as the phases of the current $i_{i}$, and the zero-crossing point can be represented by the synchronization signal PWM3 (Pulse Width Modulation). Figure 4 shows the identical wave of the synchronization control. In Figure 4, the triangle wave indicates the time-base counter register (TBCTR) of the digital signal processor (DSP). $S_{\mathrm{d}}$ denotes the phase detection signal. The signal $S_{\mathrm{d}}$ is generated

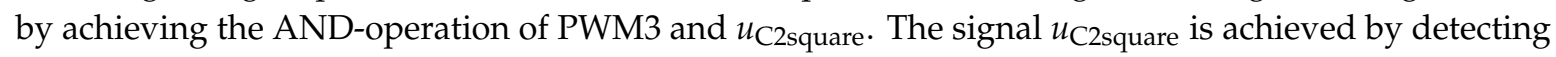
the voltage $u_{\mathrm{C} 2}$ of the capacitor $C_{2}$ and then modulating the $u_{\mathrm{C} 2}$ with a comparator to generate $u_{\mathrm{C} 2 \mathrm{square}}$. The voltage $u_{2}$ is modulated by the four compared registers 1CMPA, 1CMPB, 2CMPA, and 2CMPB in the DSP. When the four compared registers 1CMPA, 1CMPB, 2CMPA, and 2CMPB subtract or add a phase value, the voltage $u_{2}$ is modulated to shift left or right, respectively, and finally be the same phase as the resonant current $i_{2}$. The PWM3 generated by the DSP is the same phase as the voltage $u_{2}$. When each active-bridge rectifier in the MRWPT system reaches its synchronization state, that is the voltage $u_{2}$ is the same phase as the resonant current $i_{2}$, the PWM3 is the same phase as the currents $i_{i}$. In this synchronization state, the 1-state of PWM3 indicates that the $i_{i}$ is higher than zero, and the 0 -state of PWM3 indicates that the $i_{i}$ is lower than zero. Based on the method given in Liu et al. [21], when the voltage $u_{2}$ and $i_{2}$ are at the same phase in Figure 4 , the value satisfies $\theta=0^{\circ}$ and the $d=90^{\circ}$, and the phase of PWM3 represents the phase of the zero-crossing current point. When the $d$ is not equal to $90^{\circ}$, the system manipulates the phase of $U_{i}$ by tuning the turn-on sequences of $Q_{i 1}-Q_{i 4}$ until $\theta=0$.

Figure 5 shows the overall block diagram of the TS-MRWPT system. The control block in Figure 5 shows the information processing and drive signal generation. The "LPF" in Figure 5 denotes the low-pass filter block and modulates the $S_{\mathrm{d}}$ signal into a smooth DC voltage. The signal PWM3 produced by the controllers at the receivers represents the phase signal of the voltage $u_{i}$ at the receiver side. After the synchronization control of the active-bridge rectifier, the currents $i_{i}$ of the rectifiers are in phase with the voltage $u_{i}$ and the PWM 3 can represent the phase of the current $i_{i}$. Using an AND operation on both the signal PWM3 and the $Q^{\prime}{ }_{i 5}$ signal, the cyclic control of the TS-MRWPT system can turn off the $S_{i 5}$ when the current $i_{i}$ reaches its zero-crossing condition. 


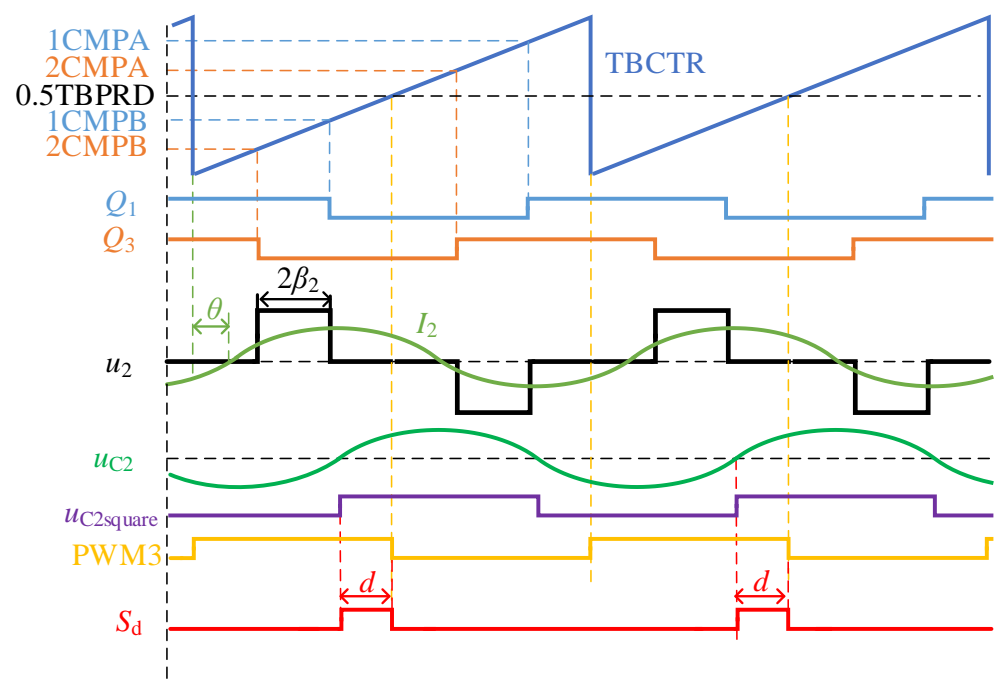

Figure 4. Generation of the synchronization control signal. CMP: compare register; PWM: Pulse Width Modulation; TBCTR: time-base counter register; TBPRD: time-base period register.

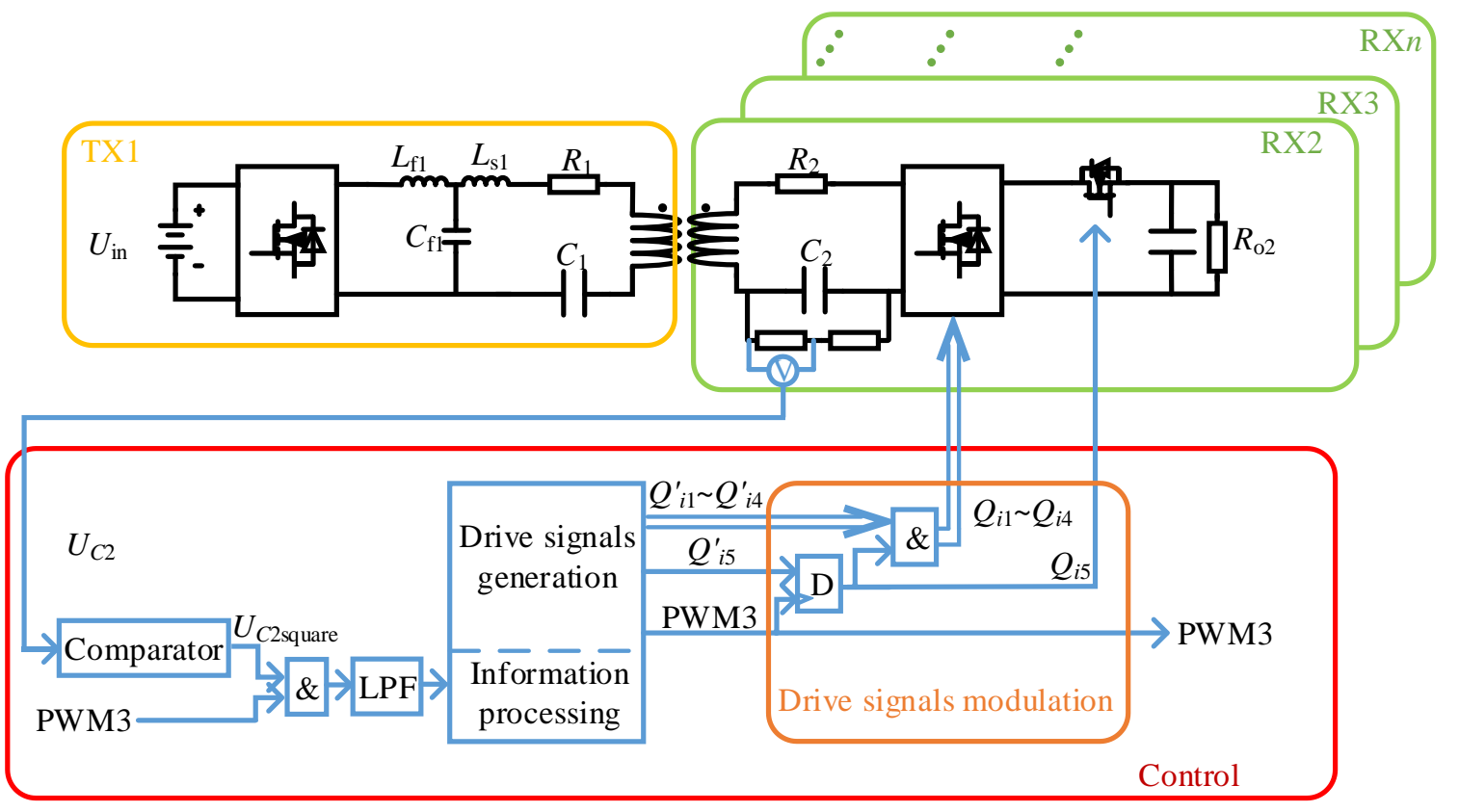

Figure 5. Overall block diagram of a time-sharing, multiple-receiver, wireless power transfer system.

LPF: low-pass filter.

In Figure 6, the drive signal $Q_{i 1}-Q_{i 4}$ must be set to a low level when the $D_{i}$ is at a low level. This is because the current $i_{i}$ still follows if the drive signal $Q_{i 1}-Q_{i 4}$ are not set to a low level. Figure 6 shows the drive signals of the active-bridge rectifiers and $S_{i 5} . Q_{i 1}-Q_{i 4}$ represent the drive signals of the phase-shifting control signals, and $Q_{i 5}$ represents the drive signal of $S_{i 5}$. During the range $t_{1}-t_{2}$, though the $Q_{i 5}$ is set to a low level, the $Q_{i 1}$ and $Q_{i 3}$ are set to a high level, and the current still follows from $S_{i 1}$ to $S_{i 3}$ and the current $i_{i}$ still follows. For the same reason, during the range $t_{3}-t_{4}$ the $Q_{i 2}$ and $Q_{i 4}$ are set to a high level, the current $I_{i}$ still follows from $S_{i 2}$ to $S_{i 4}$. Therefore, the system achieves the AND-operation of both the synchronization signal and the $Q_{i 1}-Q_{i 4}$ signal to avoid the current freewheeling. 


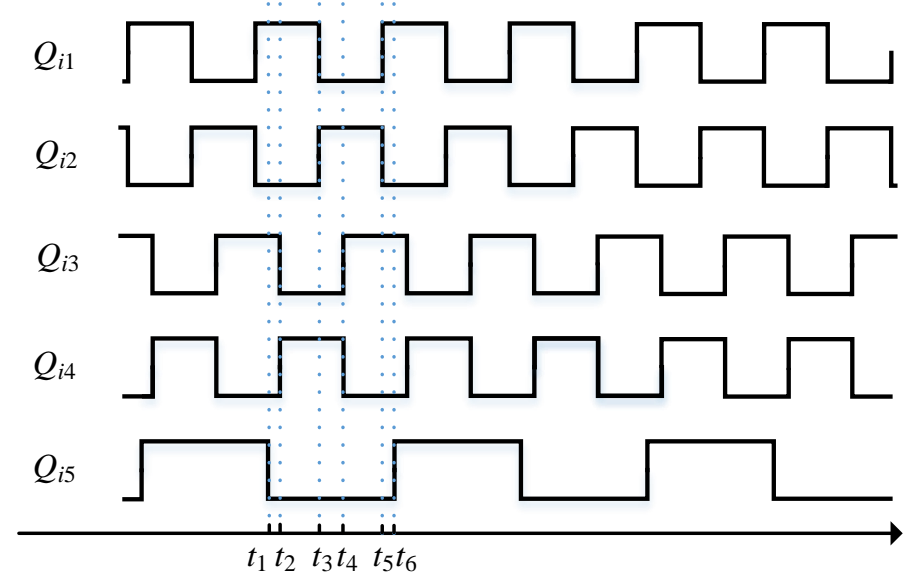

Figure 6. Drive signals of active rectifier bridges and power switch $S_{5}$.

Figure 7 displays the drive signals $Q_{i 5}$ of the time-sharing, three-receiver WPT systems. Since the D-flip-flop in Figure 5 operates when the rising edge of PWM3 arrives, there are phase-shifts in the actual $Q_{i 5}$. In Figure 7, all the actual $Q_{i 5}$ turns on in different time intervals. To reduce the complexity of the drive signals, the drive signal modulation block is also generated through the digital signal processor (DSP).

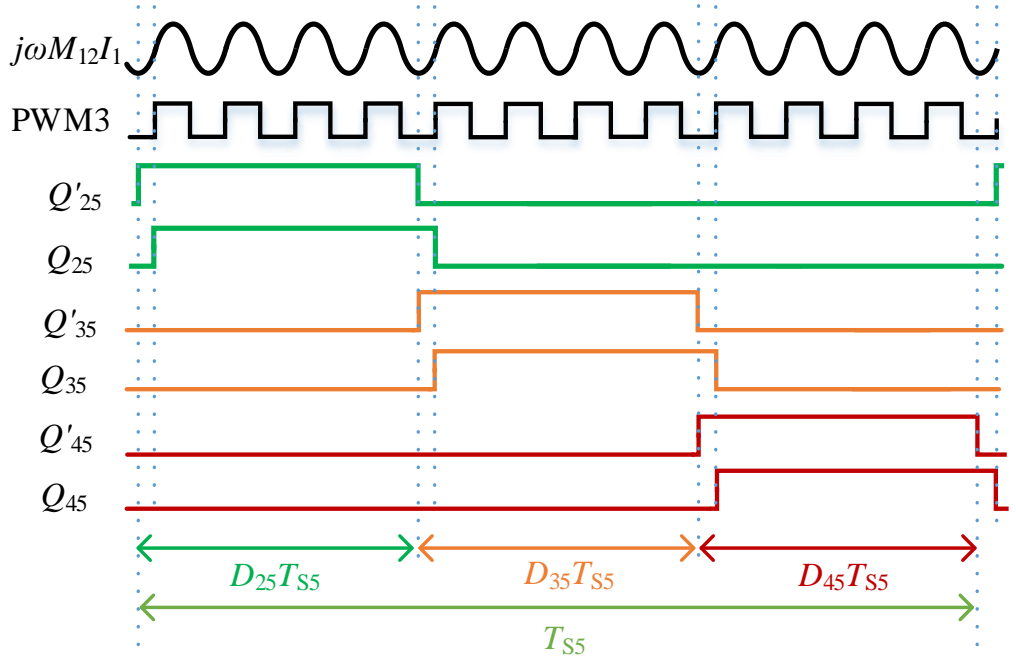

Figure 7. The actual drive signals $Q_{i 5}$ of the time-sharing, three-receiver, wireless power transfer system before and after the D-flip-flop with a rising edge trigger.

Figure 8 indicates the operating modes of the proposed TS-MRWPT system and Figure 9 shows the typical waveforms of a single-transmitter, three-receiver, time-sharing WPT system using the cyclic control method. The electrical circuits at the secondary side respectively undergo two kinds of turn-on modes and two kinds of turn-off modes within a period, as showed in Figure 8. In Figure 9, the receivers stagger their turn-on time intervals and there are direct current offsets in the voltages $u_{i \mathrm{AB}}$ because the $S_{i 5}$ turn-off and the voltage $u_{C i}$ remain the voltage before the $S_{i 5}$ turn-off. The detailed analysis of the different modes is given in the following text.

(1) Mode 1: Figure 8a displays the operating mode when $i_{i}>0(i=2,3, \ldots, n)$ and $Q_{i 5}$ are set to a high level. The $S_{i 5}$ are turned on and the potential difference $u_{\mathrm{AC}}$ is the turn-on voltage drop of the $S_{i 5}$. The current flow is $L_{i}-C_{i}-D_{i 1}-S_{i 5}-R_{\mathrm{o} i}\left(C_{\mathrm{o} i}\right)-D_{i 4}-R_{i}$. The filter capacitance $C_{\mathrm{o} i}$ are charging and the output voltage $U_{\mathrm{o} i}$ of the filter capacitance $C_{\mathrm{o} i}$ are increasing. The voltage $u_{\mathrm{C} i}(t)$ 
satisfies Equation (16). $i_{i}(t)$ and $u_{C i}(t)$ denote the instantaneous values of the resonant currents and voltages of the capacitances $C_{i}$ in Figure 8.

$$
u_{C i}(t)=\frac{i_{i}(t)}{j \omega C_{i}}
$$

(2) Mode 2: Figure 8b displays the operating mode when $i_{i}=0(i=2,3, \ldots, n)$ and $Q_{i 5}$ is set to a low level. The $S_{i 5}$ are turned off and the circuit of the receiver coils are open circuits. The potential difference is $u_{\mathrm{AC}}=u_{\mathrm{AB}}-u_{\mathrm{o} i}$, the current $i_{\mathrm{o} i}$ is supported by the filter capacitance $C_{\mathrm{o} i}$, and the filter capacitance $C_{\mathrm{o} i}$ is discharging. The current flow is $C_{\mathrm{o} i}-R_{\mathrm{o} i}$. For the currents $i_{i}=0$, the potential difference $u_{\mathrm{AB}}$ is no longer equal to the resonant voltage and the capacitance voltage $u_{C i}$ remain at the voltage $u_{C i}\left(t_{\text {model }}{ }^{-}\right)$. Therefore, the $u_{C i}$ in mode 2 in Equation (17) remains unchanged and is equal to the value of $u_{C i}\left(t_{\text {model }}{ }^{-}\right)$, which is the voltage of the $C_{i}$ at the end of mode 1 . Moreover, the $u_{\mathrm{C} i}\left(t_{\mathrm{mode}}{ }^{-}\right)$can be derived to give Equation (17) and the $\omega$ is the resonant angle frequency of the system, which is constant. The voltage $u_{\mathrm{AB}}$ satisfies Equation (18). $i_{\mathrm{p} 1}(t)$ and $u_{\mathrm{AB}}(t)$ denote the instantaneous current of the current $i_{\mathrm{p} 1}$ in Figure 3 and the instantaneous potential difference of points $\mathrm{A}$ and B in Figure 8. Furthermore, $t_{\text {mode } i}{ }^{-}$denotes the time when mode $i$ ends.

$$
\begin{gathered}
u_{\mathrm{Ci}}(t)=u_{\mathrm{C} i}\left(t_{\mathrm{mode1}}{ }^{-}\right)=\frac{i_{i}\left(t_{\mathrm{mode}^{-}}\right)}{j \omega C_{i}} \\
u_{\mathrm{AB}}(t)=j \omega M_{1 i} i_{\mathrm{p} 1}(t)+u_{C i}(t)=j \omega M_{1 i} i_{\mathrm{p} 1}(t)+\frac{i_{i}\left(t_{\mathrm{model}^{-}}\right)}{j \omega C_{i}}
\end{gathered}
$$

(3) Mode 3: Figure 8c displays the operating mode when $i_{i}<0(i=2,3, \ldots, n)$ and $Q_{i 5}$ are set to a high level. The $S_{i 5}$ are turned on and the potential difference $u_{\mathrm{AC}}$ is the turn-on voltage drop of $S_{i 5}$. The current flow is $L_{i}-C_{i}-D_{i 3}-S_{i 5}-R_{\mathrm{o} i}\left(C_{\mathrm{o} i}\right)-D_{i 3}-R_{i}$. The filter capacitance $C_{\mathrm{o} i}$ are charging and the voltage $u_{\mathrm{o} i}$ of the filter capacity $C_{\mathrm{o} i}$ are increasing. The voltage $u_{\mathrm{C} i}$ satisfies Equation (16).

(4) Mode 4: Figure 8d displays the operating mode when $i_{i}=0(i=2,3, \ldots, n)$ and $Q_{i 5}$ are set to a low level. The $S_{i 5}$ are turned off and the circuit of the receiver coils are open circuits. The potential difference is $u_{\mathrm{AC}}=u_{\mathrm{AB}}-u_{\mathrm{o} i}$, the current $i_{\mathrm{o} i}$ are supported by the filter capacity $C_{\mathrm{o} i}$, and the filter capacity $C_{\mathrm{o} i}$ are discharging. The current flow is $C_{\mathrm{o} i}-R_{\mathrm{o} i}$. For the currents $i_{i}=0$, the voltage $u_{\mathrm{C} i}$ satisfies Equations (19) and (20):

$$
\begin{gathered}
u_{C i}(t)=u_{C i}\left(t_{\text {mode } 3}{ }^{-}\right)=\frac{i_{i}\left(t_{\text {mode }^{-}}\right)}{j \omega C_{i}} \\
u_{\mathrm{AB}}(t)=j \omega M_{1 i} i_{\mathrm{p} 1}(t)+u_{C i}(t)=j \omega M_{1 i} i_{\mathrm{p} 1}(t)+\frac{i_{i}\left(t_{\text {mode } \left.^{-}\right)}\right.}{j \omega C_{i}}
\end{gathered}
$$

Figure 10 shows the general flowchart of the system control program of a TS-MRWPT system. Before the system is launched, an MRWPT system should be tuned to be under the resonant condition, and the suitable $L_{i}$ and $C_{i}$ must be designed for the resonant condition. Furthermore, the $L_{\mathrm{f} 1}, L_{\mathrm{s} 1}$, and $C_{\mathrm{f} 1}$ must be designed based on Equation (5) for an appropriate ratio $\alpha$. The $\beta_{i}$ and $D_{i}$ must be appropriately designed based on Equations (10) and (14) to satisfy both the best matching resistances and constant output voltages of each receiver before the system is launched. After the system is launched, the $S_{i 5}$ turn on and system is under synchronization control. Lastly, after the synchronization, the synchronization signal PWM3 is provided to the DSP for time-sharing control. 


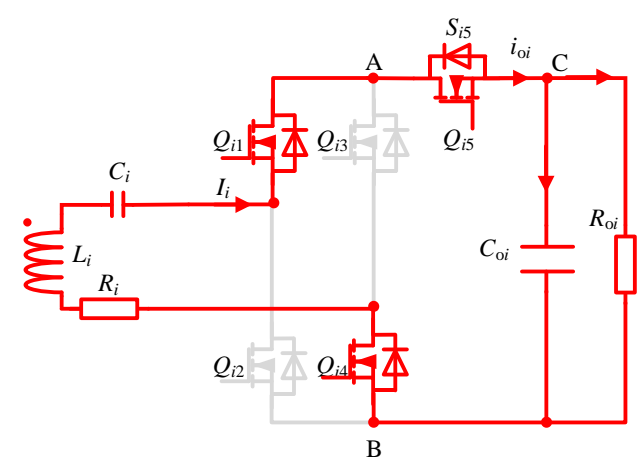

(a)

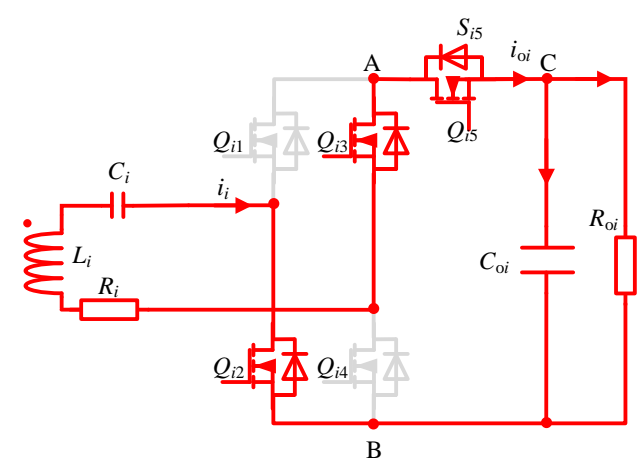

(c)

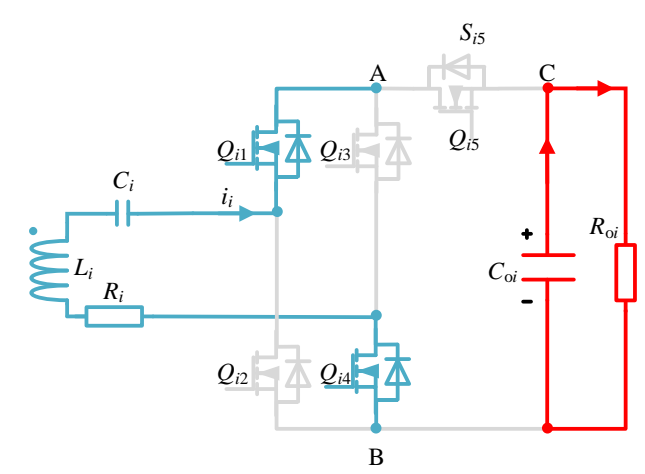

(b)

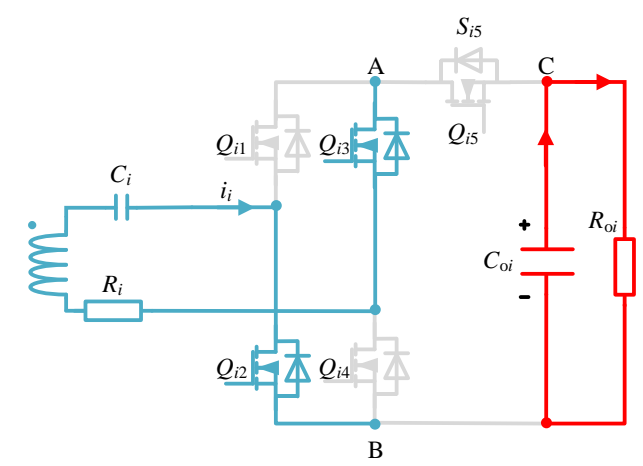

(d)

Figure 8. Operating modes of the proposed time-sharing, multiple-receiver, wireless power transfer system, where the red lines show the current loop and the blue lines show the voltage loop of $u_{\mathrm{AB}}$ : (a) mode 1, (b) mode 2, (c) mode 3, and (d) mode 4.

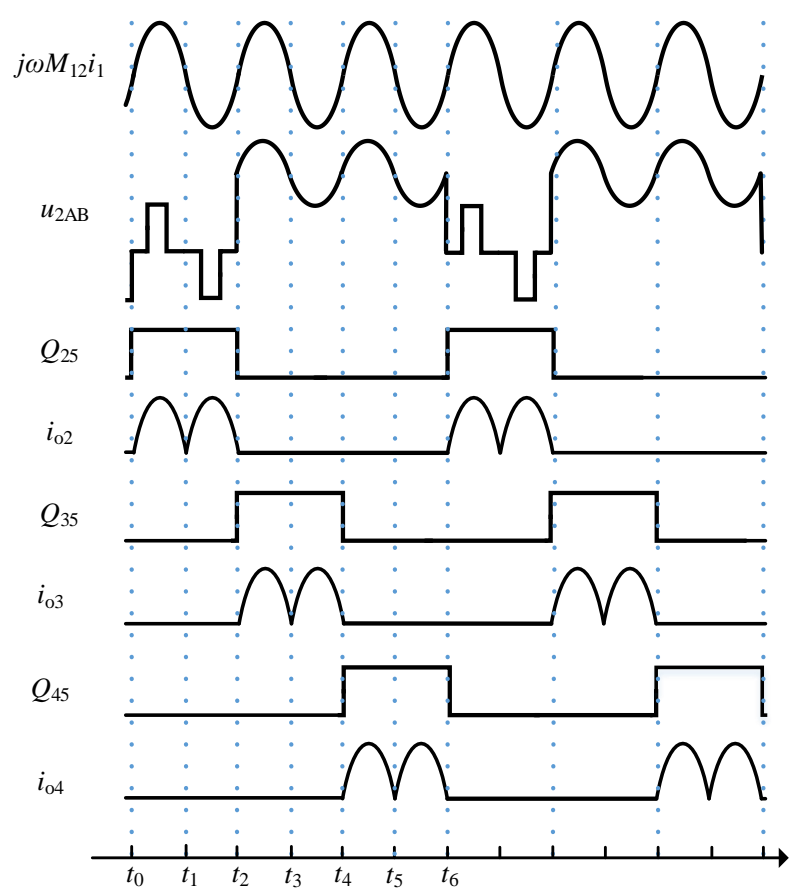

Figure 9. Typical waveforms of the proposed single-transmitter, three-receiver, time-sharing WPT system with a cyclic control method. 


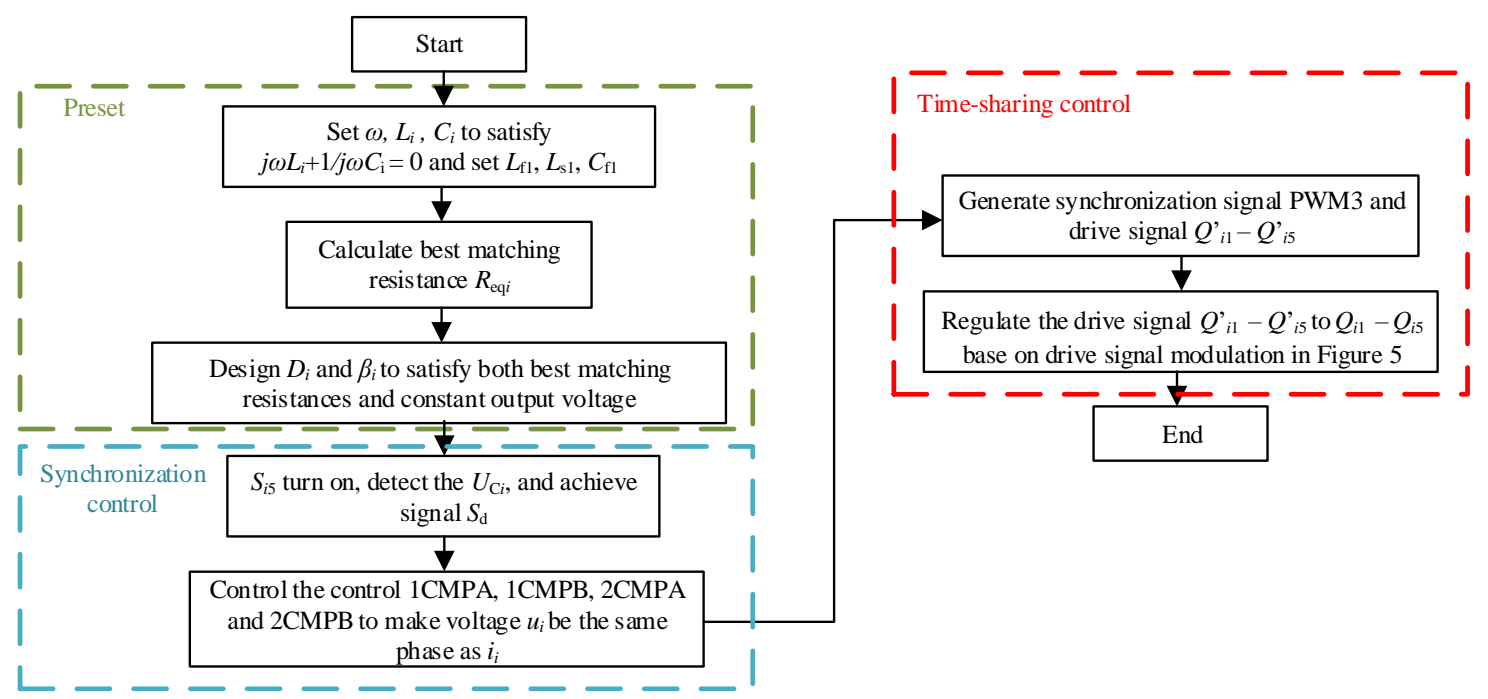

Figure 10. Flowchart of system control program.

\subsection{RC Absorbing Circuits for Time-Sharing, Multiple-Receiver Systems}

For applications, power switches are paralleled with RC absorbing circuits to absorb the peak voltage of the power switches. Therefore, as shown in Figure 11, when $Q_{i 5}$ and $Q_{i 1}-Q_{i 4}$ turn off, the current $i_{i}$ is not totally shut off, which follows through the RC absorbing circuits and brings a ringing voltage effect on $u_{i}$ and $i_{i}$. However, the capacitors of the RC absorbing circuit are small enough and the current $i_{i}$ are negligible.

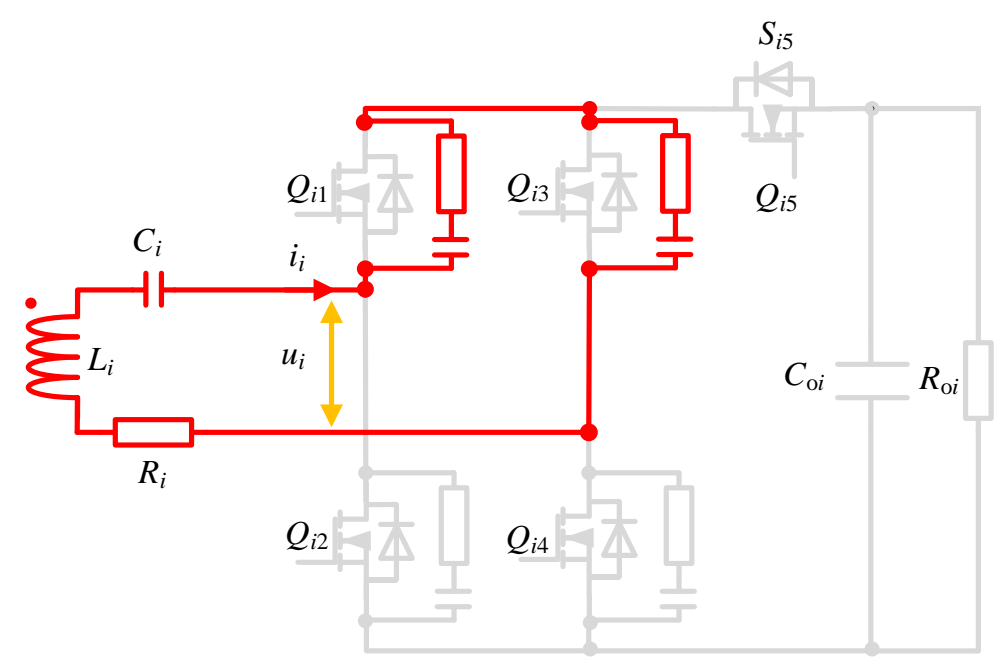

Figure 11. Current flow figure when $S_{i 5}$ turns off.

\subsection{Output Voltage Control for Time-Sharing, Multiple-Receiver, Wireless Power Transfer Systems}

In this section, this paper presents verifications that the proposed TS-MRWPT system has the advantage of both controlling the output voltages and operating under a resistance-matching condition simultaneously.

To control each receiver such that they provide different voltage levels, there are two methods used to manipulate the TS-MRWPT system. One is to control the inverter voltage $U_{1}$ for each receiver when the $S_{i 5}$ turns on, while the other is to keep the inverter voltage $U_{1}$ unchanged and tune the receiver circuit to control the output voltages of each receiver circuit. For the first method, when the power switch $S_{i 5}$ of the $i$ th receiver turns on, the inverter voltage $U_{1}$ is controlled to tune the output 
voltage. However, in the TS-MRWPT system, the method of controlling the inverter voltage $U_{1}$ to provide the target output voltage for each receiver requires building the effective synchronization between the transmitter and receivers. This adds extra complexity to the system control procedures.

Another method of controlling the receiver circuit in the proposed the TS-MRWPT system has the advantage of both controlling the output voltages and operating under a resistance-matching condition simultaneously. As seen in Equation (8), different from the buck circuits, the power switches $S_{i 5}$ have the advantages of manipulating the equivalent resistances of load resistances and hardly influencing the output voltages, and the active rectifiers are able to manipulate both the equivalent resistances of the load resistances and the output voltages. By controlling the active rectifiers and the power switch $S_{i 5}$ cooperatively, the TS-MRWPT system can both control the output voltages and operate under a resistance-matching condition simultaneously. The principal problem in a TS-MRWPT system regarding operating under a resistance-matching condition is that under the periodic control method, the duty cycle $D_{i}$ has to ensure that the number of the turn-on waveforms of each active rectifier bridge in each receiver is an integer. To operate the systems under a resistance-matching condition, the duty cycle $D_{i}$ are updated to $D_{i}{ }^{\prime}$ to ensure the number of the turn-on waveforms is a integer.

When the TS-MRWPT system satisfies $R_{i} \ll \frac{8 D_{i} R_{\mathrm{o} i} \sin ^{2} \beta_{i}}{\pi^{2}}$, by solving Equation (15), $\beta_{i}(i=2,3, \ldots, n)$ can be replaced by the output voltage $U_{\mathrm{o} i}$ :

$$
\beta_{i}=\arcsin \frac{U_{\mathrm{in}} M_{1 i} \sin \beta_{1}}{U_{\mathrm{o} i} L_{\mathrm{f} 1}}
$$

For the TS-MRWPT system, the equivalent resistance of $R_{\text {eqi }}$ in each receiver satisfies $R_{\mathrm{eq} i}=R_{\mathrm{eq} i \text {, BEST }}$ in Equation (11). By solving Equation (10), the duty cycle $D_{i}$ satisfies Equation (22):

$$
D_{i}=\frac{\pi^{2} R_{\mathrm{eq} i}}{8 R_{\mathrm{o} i} \sin ^{2} \beta_{i}}
$$

To improve the efficiency of a TS-MRWPT system, the sum of $D_{i}$ should satisfy Equation (23). When the sum of the $D_{i}$ is less than 1 , the current $I_{1}$ still follows, even though all the $S_{i 5}$ are turned off and it introduces extra power loss to the systems.

$$
\sum_{2}^{n} D_{i}=1
$$

By solving the Equations (21)-(23), the $\beta_{1}$ of the inverter at the transmitter side can be derived to give Equation (24):

$$
\beta_{1}=\arcsin \sum_{2}^{n}\left(\frac{\pi U_{\mathrm{o} i} L_{\mathrm{f} 1}}{4 U_{\mathrm{in}} M_{1 i}} \sqrt{\frac{2 R_{\mathrm{eq} i}}{R_{\mathrm{o} i}}}\right)
$$

By substituting the $\beta_{1}$ into Equations (21) and (22), the duty cycle $D_{i}$ of the power switch $S_{i 5}$ is able to control the output voltage. Moreover, the duty cycle $D_{i}$ is updated to $D_{i}{ }^{\prime}$ to ensure the number of the turn-on waveforms is a integer in Equation (25). The $[x]$ in Equation (25) is the rounding operator.

$$
D_{i}^{\prime}=\frac{\left[D_{i} N_{f}\right]}{N_{f}}
$$

Furthermore, the duty cycle $D_{i}{ }^{\prime}$ is updated to $D_{i}{ }^{\prime \prime}$ in Equation (26) to ensure that the updated duty cycle satisfies Equation (23). The function $g(x)$ is defined such that when the sum of the $D_{i}$ is less than 1 , the $D_{i}{ }^{\prime}$ that satisfies $\max \left\{D_{i}{ }^{\prime} N_{f}-\left[D_{i}{ }^{\prime} N_{f}\right]\right\}$ is previously compensated with Equation (23) until the sum of $D_{i}^{\prime \prime}$ satisfies Equation (23). 


$$
D_{i}^{\prime \prime}=g\left(D_{i}{ }^{\prime}\right)= \begin{cases}D_{i}{ }^{\prime}, & D_{i}{ }^{\prime} \text { no need to compensated } \\ \frac{D_{i}^{\prime} N_{f}+1}{N_{f}}, & D_{i}{ }^{\prime} \text { needed to compensated }\end{cases}
$$

The detailed flowchart of the output voltage control for the TS-MRWPT system is shown in Figure 12.

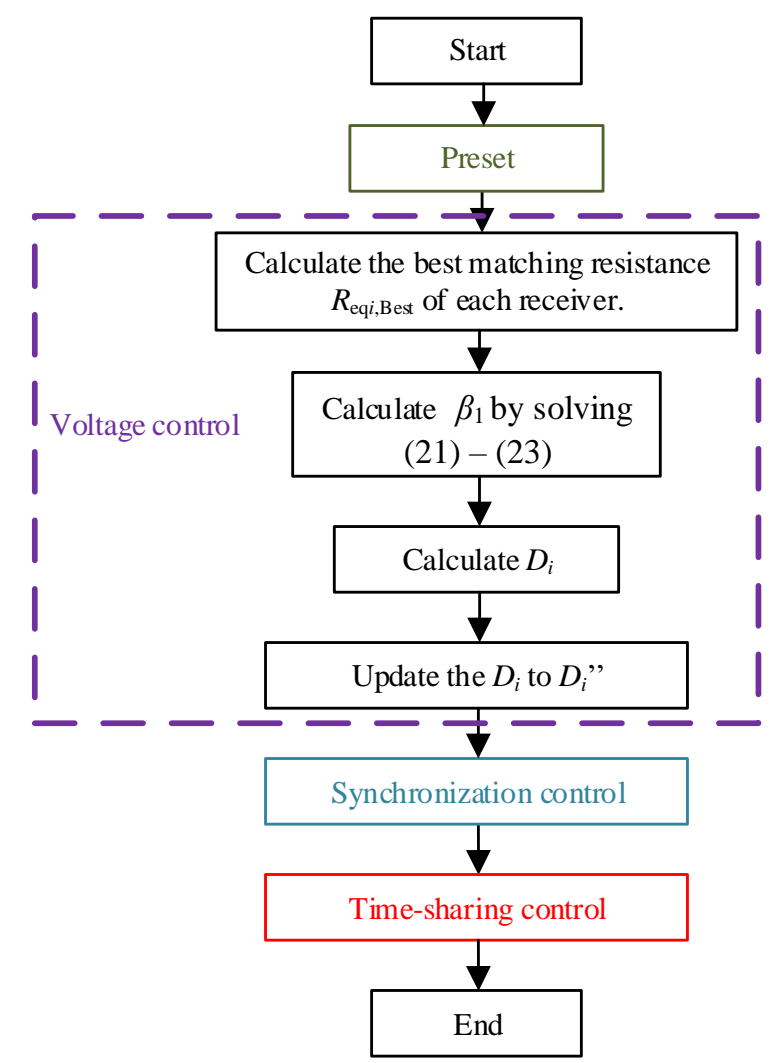

Figure 12. Flowchart of the output voltage control program for the time-sharing wireless power transfer systems.

\section{Results}

\subsection{Simulation Result of the Cross-Coupling Effect}

To verify the impact of cross-coupling between receiver coils, coil models were simulated using software COMSOL (version 5.3, COMSOL Inc., Sweden) to illustrate the cross-coupling effect in an MRWPT system. Figure 13 shows the coil models of the transmitter and receiver coils built in COMSOL. The round coil models cost a lot of computer resources in COMSOL software and will often fail to achieve convergence during simulations. Therefore, these coil models are usually constructed as near-circular coil models with a fillet as shown in Figure 13. Table 1 shows the parameters of transmitter coil and receiver coil.

Table 1. Key parameters of the coil models.

\begin{tabular}{cccccccc}
\hline Coil & Turns $\begin{array}{c}\text { Length of } \\
\text { Outer Square } \\
(\mathbf{m m})\end{array}$ & $\begin{array}{c}\text { Gap between } \\
\text { Adjacent Lines } \\
(\mathbf{m m})\end{array}$ & $\begin{array}{c}\text { Width of } \\
\text { Coil }(\mathbf{m m})\end{array}$ & $\begin{array}{c}\text { Equivalent } \\
\text { Conductivity of } \\
\text { Wire }(\mathbf{m} \cdot \mathbf{\Omega})\end{array}$ & $\begin{array}{c}\text { Equivalent } \\
\text { Resistance } \\
(\mathbf{m} \boldsymbol{\Omega})\end{array}$ & $\begin{array}{c}\text { Self-Inductance } \\
(\boldsymbol{\mu} \mathbf{H})\end{array}$ \\
\hline Transmitter coil & 5 & 420 & 2 & 2 & $1.5 \times 10^{6}$ & 17.439 & 17.535 \\
\hline Receiver coil & 8 & 200 & 2 & 2 & $1.5 \times 10^{6}$ & 15.433 & 18.103 \\
\hline
\end{tabular}




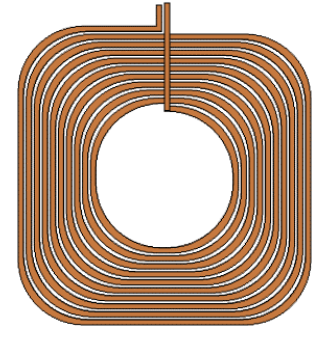

(a)

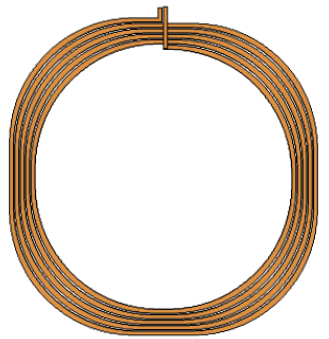

(b)

Figure 13. Simulation models of coils with a square model: (a) transmitter coil and (b) receiver coil.

The distance $d_{\text {TR }}$ between the two receiver coils and the transmitter coil was $100 \mathrm{~mm}$. The distance $d_{\mathrm{R}}$ between the centers of the receivers was $180 \mathrm{~mm}$. Figure 14a shows the magnetic field simulation of the transmitter coil, which was activated with a $1 \mathrm{~A}, 100 \mathrm{kHz}$ current. Figure $14 \mathrm{~b}$ shows the magnetic field simulation of the receiver coil 3 (Rx3), which was activated with a $1 \mathrm{~A}, 100 \mathrm{kHz}$ current. Figure $14 \mathrm{c}$ shows the magnetic field simulation of both transmitter and receiver coil 3 , which were both activated with a $1 \mathrm{~A}, 100 \mathrm{kHz}$ current. According to the simulation results, the magnetic flux densities of the center point of coil $2(\mathrm{Rx} 2)$ were $10.184 \mu \mathrm{T}, 2.9245 \mu \mathrm{T}$, and $13.024 \mu \mathrm{T}$, respectively, when the system was activated using $T \times 1, R \times 3$, and both $T \times 1$ and $R \times 3$. Comparing the results in Figure 14, the received magnetic field of receiver coil 2 was improved by the cross-coupling from receiver coil 3. However, when the systems were activated by both Tx1 and Rx3, the magnetic flux densities were improved, and the resonant conditions were weakened by the cross-coupling of the receivers. Furthermore, the induced voltage on $R \times 2$, which was generated by $I_{3}$, varied when $I_{3}$ varied. Therefore, the cross-coupling problems are great challenges for an MRWPT system.

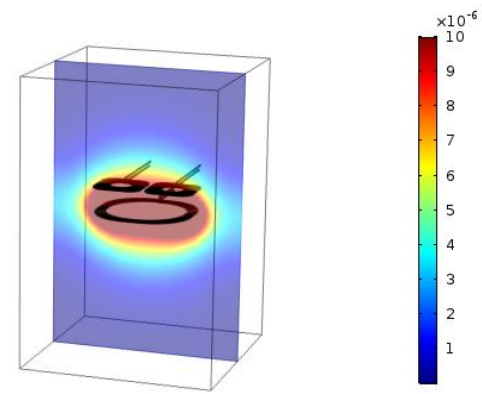

(a)

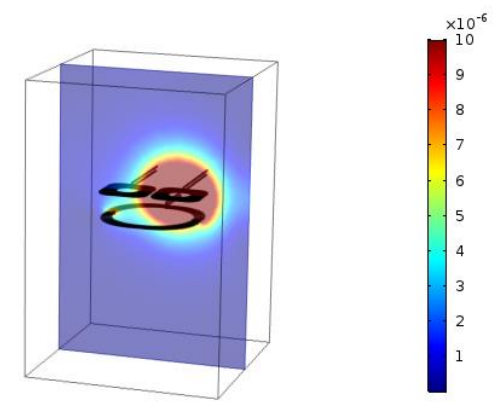

(b)

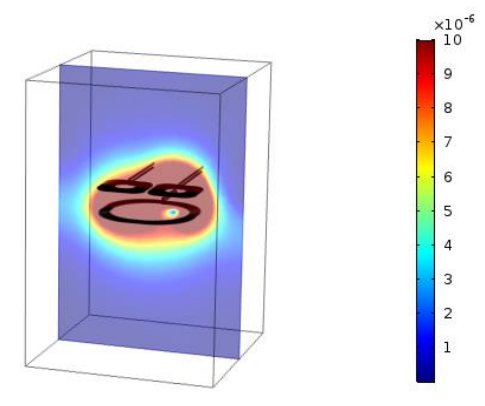

(c)

Figure 14. Simulations of the magnetic field distribution: (a) transmitter coil was activated, (b) receiver coil 3 was activated, and (c) both transmitter coil and receiver coil 3 were activated. 


\subsection{Simulation and Experimental Results of Time-Sharing, Multiple-Receiver WPT Systems}

To verify the validity of the proposed TS-MRWPT system, the mathematical model was constructed and simulated using the PLECS platform (version 4.12, Plexim GmbH Inc., Switzerland). An experimental prototype was constructed as displayed in Figure 15. Table 2 presents parameters of a time-sharing, two-receiver WPT system. The transmitter coil and receiver coils were constructed based on the COMSOL simulation model.

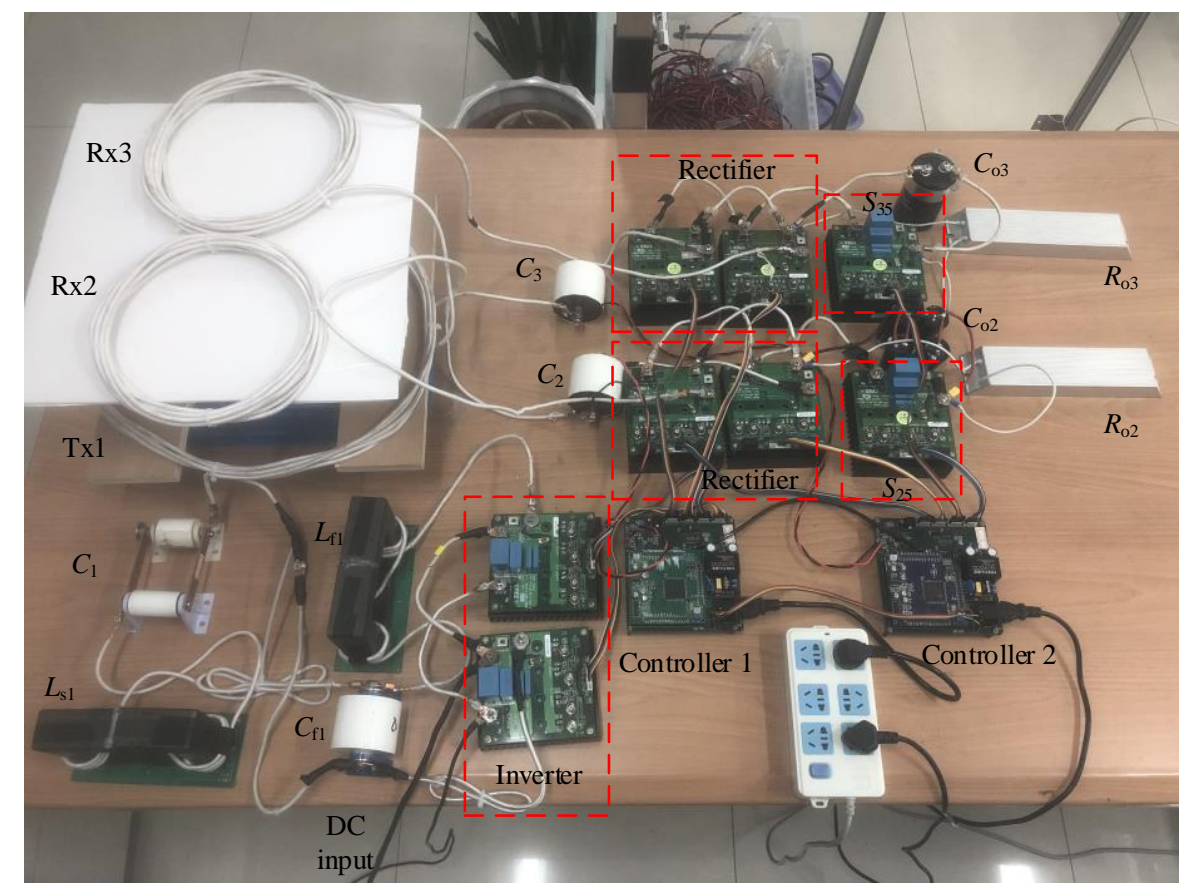

Figure 15. Experimental platform of the TS-MRWPT system.

Table 2. Key parameters of the time-sharing, two-receiver, wireless power transfer models.

\begin{tabular}{ccc}
\hline Symbol & Quantity & Value \\
\hline$L_{1}, L_{2}, L_{3}$ & Self-inductances of coils & $22.78 \mu \mathrm{H}, 24,53 \mu \mathrm{H}, 24.62 \mu \mathrm{H}$ \\
\hline$L_{\mathrm{f} 1}, L_{\mathrm{s} 1}$ & Inductances & $25.12 \mu \mathrm{H}, 25.20 \mu \mathrm{H}$ \\
\hline$C_{1}, C_{2}, C_{3}, C_{\mathrm{f} 1}$ & Compensation capacitors & $110 \mathrm{nF}, 100 \mathrm{nF}, 100 \mathrm{nF}, 100 \mathrm{nF}$ \\
\hline$C_{\mathrm{o} 2}, C_{\mathrm{o} 3}$ & Filter capacitors & $2200 \mu \mathrm{F}, 2200 \mu \mathrm{F}$ \\
\hline$R_{1}, R_{2}, R_{3}$ & Parasitic resistances of coils & $21.74 \mathrm{~m} \Omega, 17.03 \mathrm{~m} \Omega, 17.53 \mathrm{~m} \Omega$ \\
\hline$N_{1}, N_{2}, N_{3}$ & Turns of coils & $5,8,8$ \\
\hline$r_{1}, r_{2}, r_{3}$ & Radius of coils & $420 \mathrm{~mm}, 200 \mathrm{~mm}, 200 \mathrm{~mm}$ \\
\hline$R_{\mathrm{o} 2}, R_{\mathrm{O} 3}$ & Load resistances & $5 \Omega, 5 \Omega$ \\
\hline$f_{1}$ & Operating frequency & $5 \mathrm{kHz}$ \\
\hline$f_{2}$ & Operating frequency & $0.9660 \mu \mathrm{H}$ \\
\hline$M_{23}$ & Mutual inductance &
\end{tabular}

In Figure 15, Tx1 indicates the transmitter coils, $\mathrm{Rx} 2$ and $\mathrm{R} \times 3$ indicates the receiver coils. The experimental coils in Figure 15 were constructed with a round model and the measured parameters are listed in Table 2. $C_{i}$ indicates the compensation capacitances. The active bridge at each coil was constructed with two half-bridge circuit modules KIT8020CRD8FF1217P-1 (Cree Inc.). The MOSFET in the half-bridge circuit modules was SCT3030KL (Rohm Inc.), the turn-on resistance of which was about 
$30 \mathrm{~m} \Omega$. Systems were respectively controlled by controller 1 and controller 2, which each consisted of a digital signal processor (DSP) TMS320F28335 (Texas Instruments Ins.).

Figure 16 shows the experiment waveforms of the TS-WPT system with active-bridge rectifiers. The voltages in Figure 16a show the drive signals of the TS-MRWPT system, where the $Q_{21}$ and $Q_{24}$ agreed with the drive signal modulation block in Figure 5. Figure 16b shows the waveforms of the receiver coil $R \times 2$, where the ringing in $U_{2}$ was caused by the $R C$ absorbing circuit, which was parallel to the power switch. When the $S_{i 5}$ turned off, the circuit at the secondary side was cut off, but currents $I_{2}$ still followed the RC absorbing circuit. The current $I_{2}$ in Figure $16 \mathrm{~b}$ shows that the current $I_{2}$ was generally cut off when the $Q_{25}$ turned off. Figure 16c shows the capacitor voltages of the TS-MRWPT system. The $U_{C 2}$ held the voltage value when $S_{25}$ turned off, and $C_{2}$ was the resonant condition when $S_{25}$ turned on.

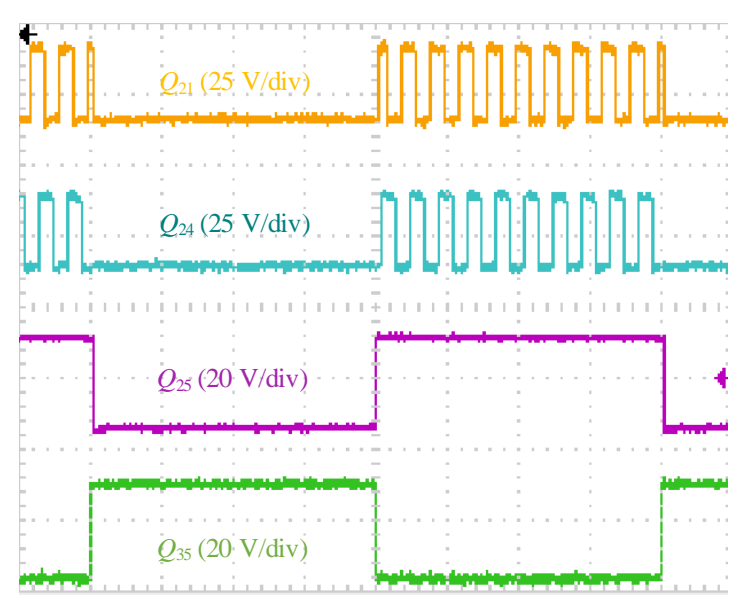

Time $(2.5 \mu \mathrm{s} / \mathrm{div})$

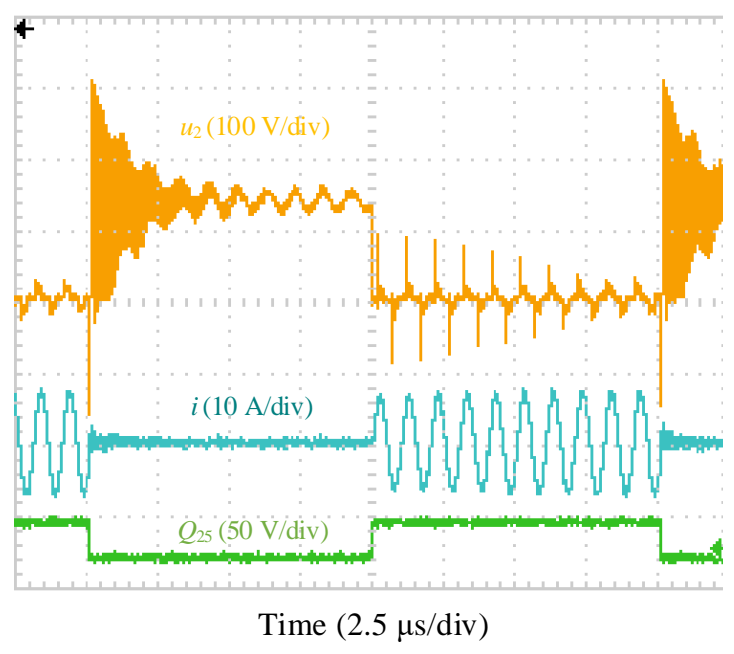

(b)

(a)

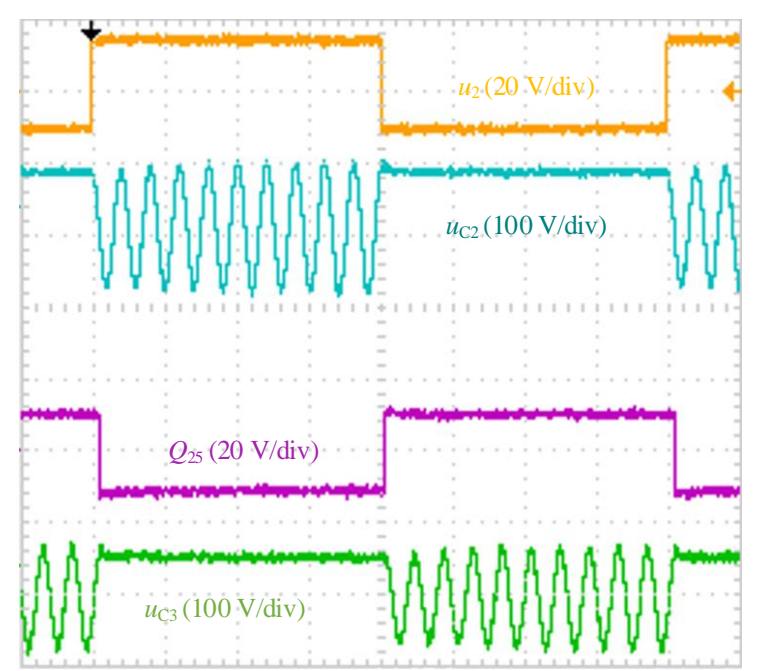

Time $(2.5 \mu \mathrm{s} / \mathrm{div})$

(c)

Figure 16. Experimental waveform: (a) drive signals waveform, (b) waveforms of coil 2, and (c) voltage waveforms of the capacitors.

Figure 17 shows the simulation waveform comparison of the TS-MRCT system without the RC absorbing circuits and with the RC absorbing circuits. In Figure 17a, the TS-MRWPT system without 
the RC absorbing circuits nearly removed the ringing effect, while the TS-MRWPT system with the RC absorbing circuits had a ringing effect. Figure $17 \mathrm{c}$,d shows the enlarged view of the voltage $U_{2}$ and current $I_{2}$ in the TS-MRWPT system with RC absorbing circuits and the ringing effect was weakened by improving the resistances of the RC absorbing circuit from $10 \Omega$ to $100 \Omega$.
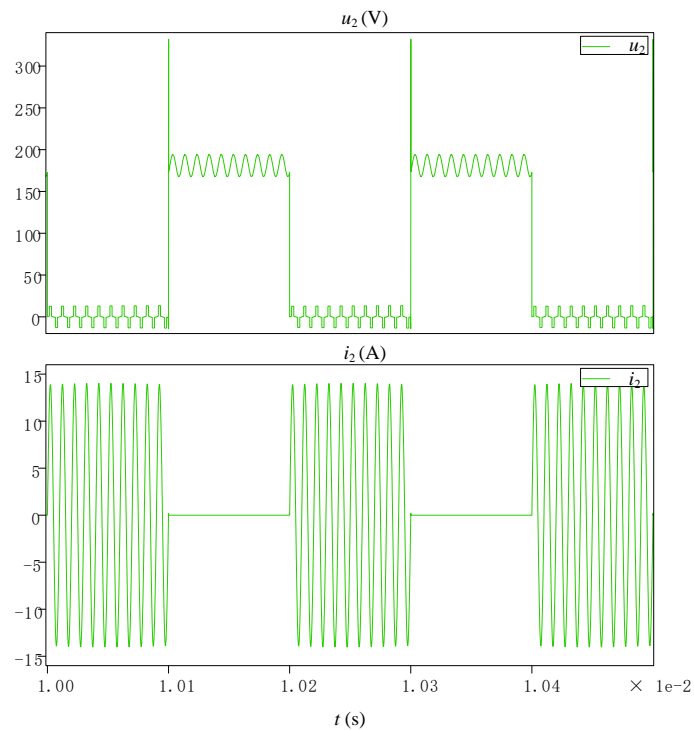

(a)
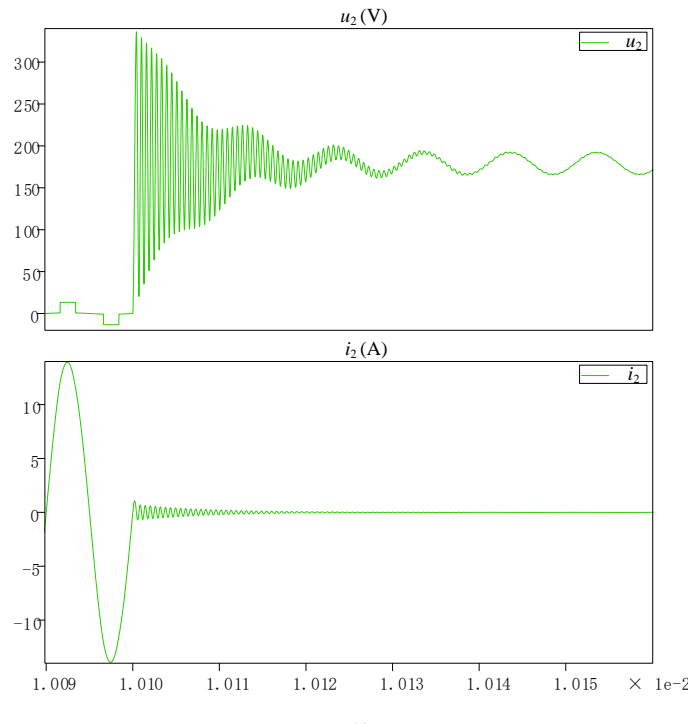

(c)
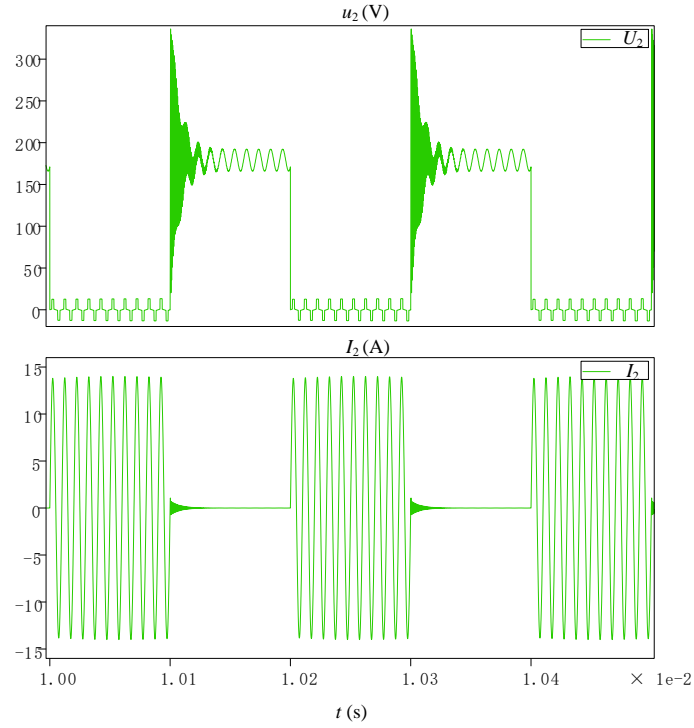

(b)

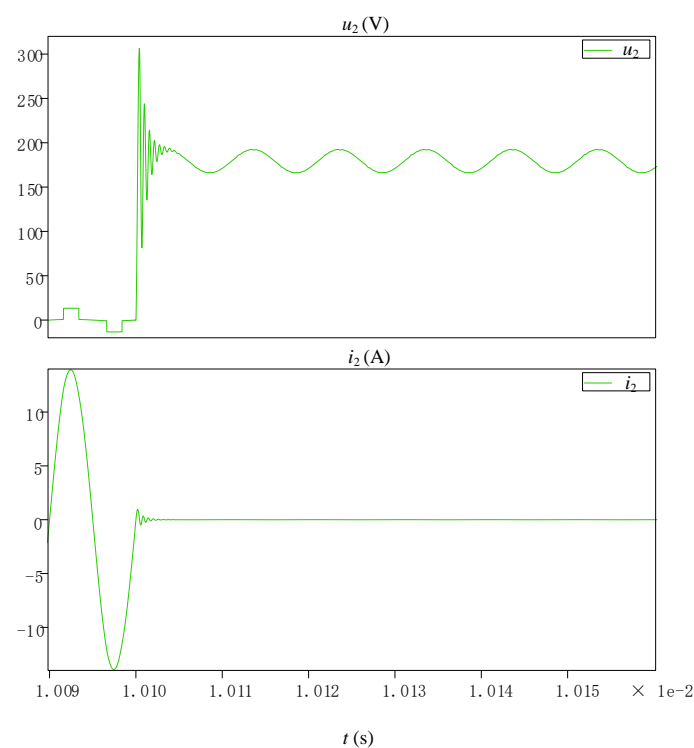

(d)

Figure 17. Simulation waveform: (a) system without RC absorbing circuits; (b) system with RC absorbing circuit, where the resistance was $10 \Omega$; (c) enlarged view of the voltages and currents of the system with RC absorbing circuits, where the resistances were $10 \Omega$; and (d) enlarged view of the voltages and currents of system with two RC absorbing circuits, where the resistances were $100 \Omega$.

To verify the validity of the TS-MRWPT system, the TS-MRWPT system was compared to a MRWPT system that had the same parameters and under a best-matching resistance condition based on Equation (11). The parameters of the TS-MRWPT system and the MRWPT system follow Table 2, and the power switches $S_{i 5}$ in the MRWPT system were removed to avoid the extra power loss from $S_{i 5}$. Figure 18a shows the efficiency comparisons of different topologies and control strategies. Figure 18a shows that the simulation efficiencies of the MRWPT system without cross-coupling were slightly 
higher than the simulation efficiencies of the TS-MRWPT system, and the simulation efficiencies of the MRWPT system with cross-coupling was severely weakened by the cross-coupling effect and the transfer efficiencies were lower than $30 \%$. The experimental results of the proposed TS-MRWPT system achieved a maximum efficiency of about $82.4 \%$ at the distance of $50 \mathrm{~mm}$, and decreased due to the increasing distances between the transmitter and receivers. Figure $18 \mathrm{c}$ shows the same tendency with Figure 18a, where the voltage $U_{\mathrm{o} 2}$ was improved in the TS-MRWPT system. This shows that the proposed TS-MRWPT reduced the cross-coupling effect between receivers and increased the efficiencies of the MRWPT systems.

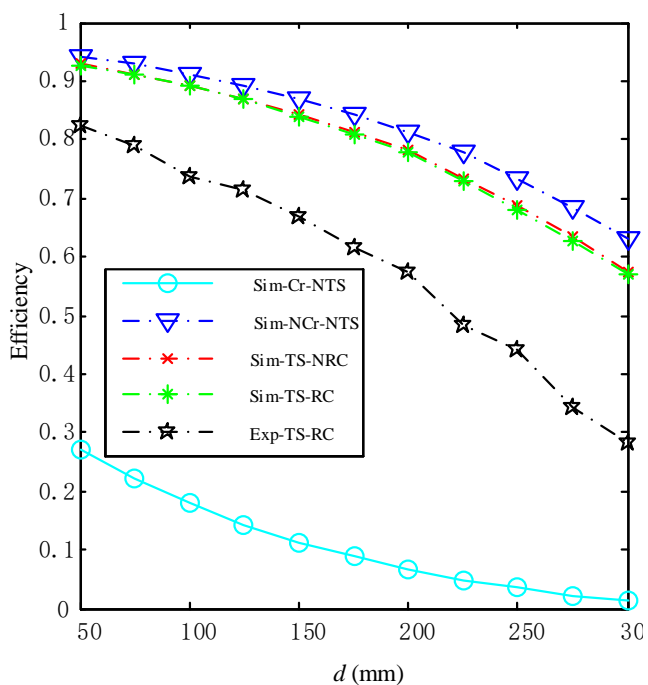

(a)

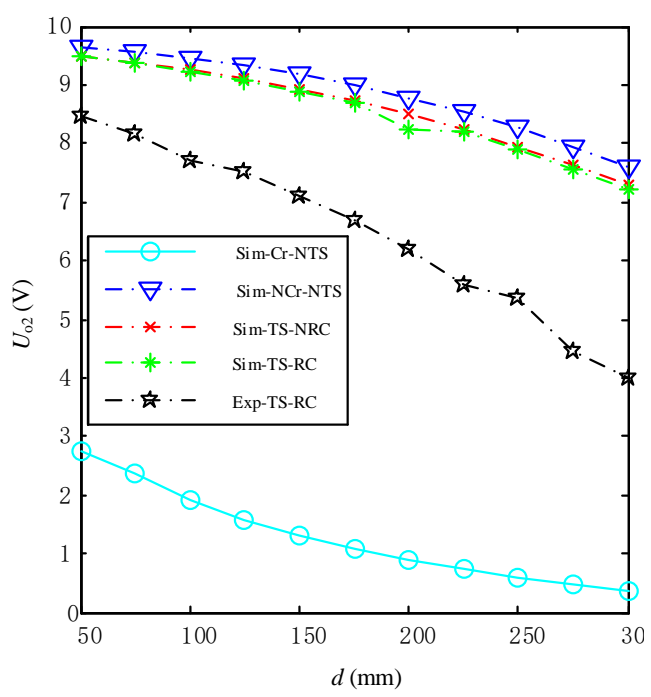

(c)

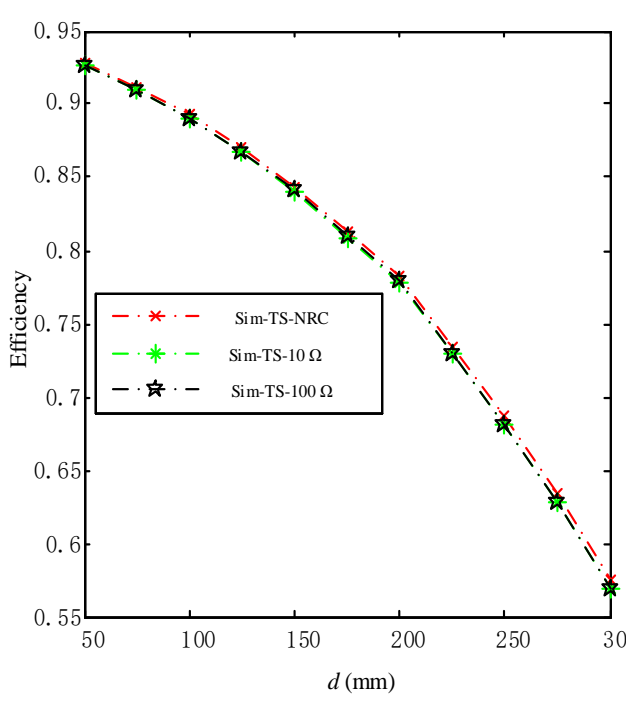

(b)

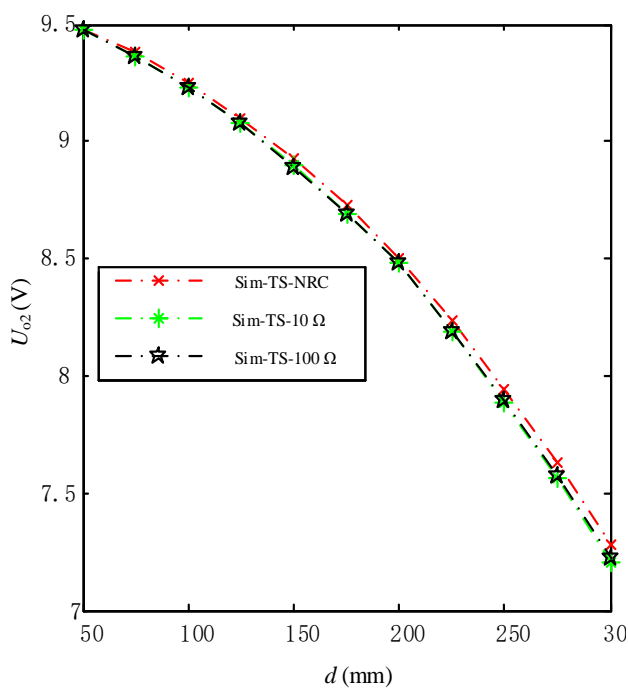

(d)

Figure 18. Simulation and experiment results: "Sim" and "Exp" indicates simulation and experiment, respectively. "Cr" and "NCr" indicates the simulation setup with cross-coupling and without cross-coupling, respectively. "TS" and "NTS" indicates the system having time-sharing control and without time-sharing control, respectively. "NRC" and "RC" indicates the system introducing an RC absorbing circuit and without an RC absorbing circuit, respectively. " $10 \Omega$ " and " $100 \Omega$ " indicates the absorbing resistances. (a) Efficiency comparisons. (b) Efficiency comparisons between systems with RC absorbing resistances and without RC resistances. (c) Output voltage $U_{\mathrm{o} 2}$ comparisons. (d) Output voltage $U_{\mathrm{o} 2}$ comparisons between systems with $\mathrm{RC}$ absorbing resistances and without RC absorbing resistances. 
In Figure 18a,c, the simulation efficiencies and output voltages of the MRWPT system without a cross-coupling effect was higher than the ones of the TS-MRWPT. This was because the MRWPT system without a cross-coupling effect was built without power switches $S_{i 5}$, and hence without the power loss. However, in a practical application, the MRWPT system without a cross-coupling effect is an ideal model and cannot be achieved if the receiver coil gets close. Furthermore, the experimental efficiency of the TS-MRWPT was about 10\% lower than the simulation efficiency of the TS-MRWPT. This was because the resistance of the inductances and capacitances of the LCL compensation topologies were not considered in the simulation, and the parameter errors of the components in the compensation network also introduced an extra efficiency decrease to the TS-MRWPT systems. Both the simulation efficiencies and experimental efficiencies of the TS-MRWPT system decreased when the distance between the transmitter coil and the receiver coil got larger (Figure 18a).

Figure 18b,d shows that the simulation comparisons of the efficiencies and output voltage $U_{\mathrm{o} 2}$ for systems with $\mathrm{RC}$ absorbing circuits and without $\mathrm{RC}$ absorbing circuits. The efficiencies and voltage $U_{\mathrm{o} 2}$ of the circuits with RC absorbing circuits and without absorbing were nearly the same. The simulation results showed that the efficiencies of the TS-MRWPT system with an RC absorbing circuit were a little higher than the circuit without RC absorbing circuits, and the efficiencies of an RC absorbing circuit with an absorbing resistance $100 \Omega$ was a bit higher than the efficiencies with absorbing resistance of $10 \Omega$. This verifies that a higher absorbing resistance helped to reduce the ringing effect of the TS-MRWPT systems and improve the system efficiency.

\subsection{Simulation Comparison between the Time-Sharing Method and Compensation Method}

To verify the validity of the time-sharing voltage control for the TS-MRWPT system, the system was compared with the efficiency of the compensation method in Fu et al. [25]. Table 3 lists the comparison result of the two different approaches. The comparisons were constructed under the parameters listed in Table 2. Based on Fu et al. [25], the compensation capacitances $C_{2}$ and $C_{3}$ in the compensation method were set to $110 \mathrm{nF}$ and $100 \mathrm{nF}$, respectively, to compensate for the cross-coupling inductances between receivers when $d_{\mathrm{TR}}=50 \mathrm{~mm}$. The maximum simulation efficiency of the cross-coupling method was about $94.3 \%$, which was slightly higher than the maximum simulation efficiency of the time-sharing method, which was about 93.2\%. In Figure 19, the distance $d_{\mathrm{TR}}$ between the transmission coil and receiving coil was swept from $50 \mathrm{~mm}$ to $300 \mathrm{~mm}$ and the efficiencies of the system using the compensation method, which are marked "Sim-Com-NTS", were similar to the efficiencies of the system without cross-coupling in Figure 19. The compensation method had a better performance than the time-sharing method when the relative distance between the receiver coils were unchanged. However, the compensation method must operate under fixed currents and the efficiencies dropped rapidly when the load resistances and cross-coupling changed. The simulation comparisons regarding the efficiency are discussed in later sections.

Comparisons were made to compare the performances of the compensation method and time-sharing method under different distances between the receiver coils in a two-receiver WPT system. Table 3 displays the parameters of the coil models. The receivers were placed as displayed in Figure 20: receiver coil 2 was placed at position " $A$ " and receiver coil 3 was placed at position " $B$ " or " $C$," transmitter coil 1 was placed under the receiver coils, and $d_{\mathrm{TR}}=100 \mathrm{~mm}$ where the mutual inductance $M_{T R}$ between the transmitter coil and receiver coil satisfied $M_{T R}=0.43343 \mu \mathrm{H}$. The position " $\mathrm{B}$ " and "C" were reached by rotating the receiver coil at place " $\mathrm{A}$ " by $90^{\circ}$ and $180^{\circ}$, respectively. The mutual inductance $M_{23}$ between the two receiver coils satisfied $M_{23}=0.072997 \mu \mathrm{H}$ when the receivers were placed at positions " $\mathrm{A}$ " and " $\mathrm{B}$," and satisfied $M_{23}=0.033368 \mu \mathrm{H}$ when the receivers were placed at positions " $\mathrm{A}$ " and " $\mathrm{C}$ ". Both the compensation and time-sharing methods were simulated under best-matching resistance conditions based on Equation (11), where the $R_{\text {eq2, BEST }}=21.74 \mathrm{~m} \Omega$ and $R_{\text {eq3, BEST }}=21.74 \mathrm{~m} \Omega$. In the compensation system, the derived compensation capacitance $C_{2}=C_{3}$ $=0.507821 \mu \mathrm{F}$ when receiver coil 2 was placed at position " $\mathrm{A}$ " and receiver coil 3 was placed at " $\mathrm{B}$ " were directly applied to systems where receiver coil 2 was placed at " $\mathrm{A}$ " and receiver coil 3 was 
placed at "C." Table 4 displays the simulation result. In Table 4 , the compensation method had a higher system efficiency than the time-sharing method when the cross-coupling was compensated. However, when receiver coil 3 moved to the position " $C$," the efficiency rapidly dropped due to the variation of the mutual inductances of the cross-coupling effect. However, the time-sharing method had a more flexible choice for the placement of the receiver coil and achieved a high efficiency at position "C." The efficiency of the time-sharing method was generally unchanged despite the variation of the distance between receivers.

Table 3. Key parameters of the coil models.

\begin{tabular}{cccccccc}
\hline Coil & Turns & $\begin{array}{c}\text { Length of } \\
\text { Outer Square } \\
(\mathbf{m m})\end{array}$ & $\begin{array}{c}\text { Gap between } \\
\text { Adjacent Lines } \\
(\mathbf{m m})\end{array}$ & $\begin{array}{c}\text { Width of } \\
\text { Coil }(\mathbf{m m})\end{array}$ & $\begin{array}{c}\text { Equivalent } \\
\text { Conductivity of } \\
\text { Wire }(\mathbf{m} \cdot \mathbf{\Omega})\end{array}$ & $\begin{array}{c}\text { Equivalent } \\
\text { Resistance } \\
(\mathbf{m} \Omega)\end{array}$ & $\begin{array}{c}\text { Self-Inductance } \\
(\mu \mathrm{H})\end{array}$ \\
\hline Receiver coil & 7 & 130 & 2 & 2 & $1.5 \times 10^{6}$ & 7.4915 \\
\hline Transmitter coil & 5 & 420 & 2 & 2 & $1.5 \times 10^{6}$ & 15.433 \\
\hline
\end{tabular}

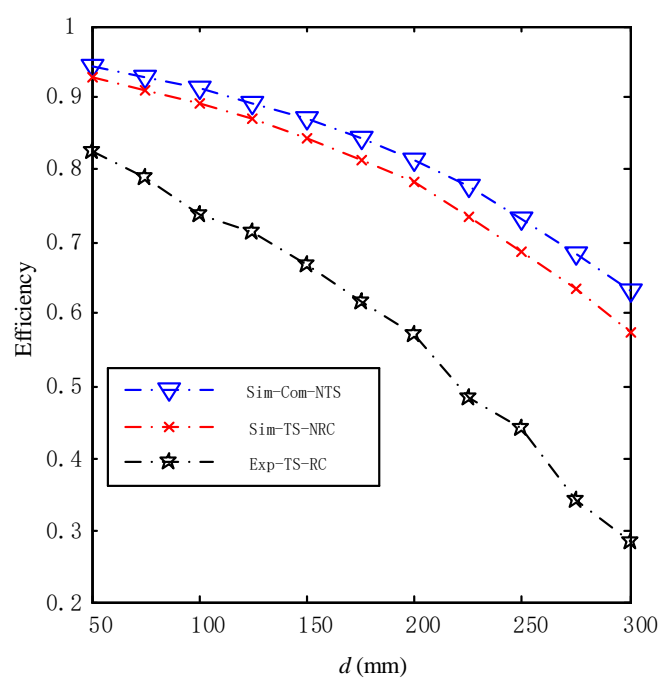

Figure 19. Simulation results of the time-sharing, three-receiver, wireless power transfer system with an output voltage control strategy.

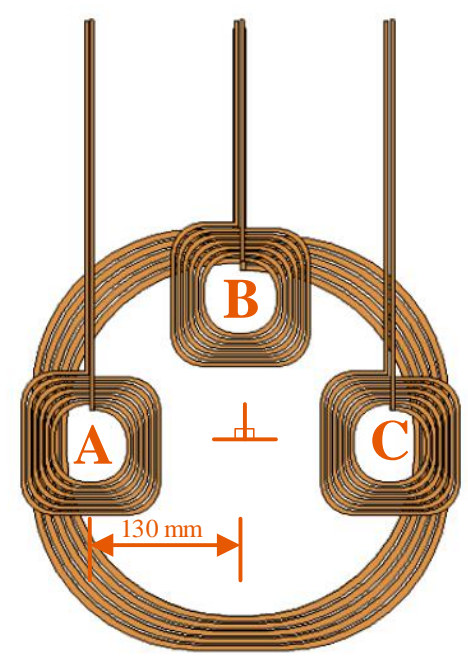

Figure 20. Simulation results of a time-sharing, three-receiver, wireless power transfer system with an output voltage control strategy. 
Table 4. Simulation results of the comparison between the time-sharing and compensation methods.

\begin{tabular}{ccc}
\hline \multirow{2}{*}{ Method } & \multicolumn{2}{c}{ Efficiency } \\
\cline { 2 - 3 } & Position “B” & Position “C” \\
\hline Cross-coupling compensation method & $88.33 \%$ & $77.66 \%$ \\
\hline Time-sharing method & $85.41 \%$ & $85.40 \%$ \\
\hline
\end{tabular}

\subsection{Simulation Result of the Voltage Control of a Time-Sharing, Multiple-Receiver WPT System}

To verify the validity of the output voltage control for the TS-MRWPT systems, a three-receiver WPT system with a time-sharing method was simulated in PLECS software. The parameters of the proposed system are shown in Table 5. The active-bridge rectifiers of the TS-MRWPT system were constructed without $R C$ absorbing circuits. The calculated $D_{2}, D_{3}$, and $D_{4}$ were $D_{2}=0.373, D_{3}=0.291$, and $D_{4}=0.336$, and the updated $D_{2}{ }^{\prime \prime}, D_{3}{ }^{\prime \prime}$, and $D_{4}{ }^{\prime \prime}$ were $D_{2}{ }^{\prime \prime}=0.35, D_{3}{ }^{\prime \prime}=0.3$, and $D_{4}{ }^{\prime \prime}=0.35$. Figure 21 shows the voltage $U_{i}$ and current $i_{i}$ of each receiver and the output voltage $U_{\mathrm{o} 2}, U_{\mathrm{o} 3}$, and $U_{\mathrm{o} 4}$ achieved $7.29 \mathrm{~V}, 8.98 \mathrm{~V}$, and $11.03 \mathrm{~V}$, respectively with target powers of $8 \mathrm{~V}, 10 \mathrm{~V}$, and $12 \mathrm{~V}$, respectively. The simulation results achieved a simulation efficiency of $89.66 \%$, and in comparison, the simulation results of the traditional MRWPT system, which compensated for the cross-coupling with resonant capacitor in Fu et al. [25] under the same system parameters achieved a simulation efficiency of $94.66 \%$. It verifies that though the efficiency was lower than the efficiency of the system that compensated the cross-coupling, the TS-MRWPT system still achieved a high efficiency. Moreover, the TS-MRPWT had the advantages that TS-MRWPT was applicable for a time-variant cross-coupling MRWPT system.

Table 5. Key parameters of the time-sharing, three-receiver, wireless power transfer models.

\begin{tabular}{ccc}
\hline Symbol & Quantity & Value \\
\hline$L_{1}, L_{2}, L_{3}, L_{4}$ & Self-inductances of coils & $22.78 \mu \mathrm{H}, 24,53 \mu \mathrm{H}, 24,53 \mu \mathrm{H}, 24,53 \mu \mathrm{H}$ \\
\hline$L_{\mathrm{f} 1}, L_{\mathrm{s} 1}$ & Inductances & $25 \mu \mathrm{H}, 25 \mu \mathrm{H}$ \\
\hline$C_{1}, C_{2}, C_{3}, C_{4}, C_{\mathrm{f} 1}$ & Compensation capacitors & $110 \mathrm{nF}, 100 \mathrm{nF}, 100 \mathrm{nF}, 100 \mathrm{nF}, 100 \mathrm{nF}$ \\
\hline$C_{\mathrm{o} 2}, C_{\mathrm{o} 3}, C_{\mathrm{o} 4}$ & Filter capacitors & $2200 \mu \mathrm{F}, 2200 \mu \mathrm{F}, 2200 \mu \mathrm{F}$ \\
\hline$R_{1}, R_{2}, R_{3}, R_{4}$ & Parasitic resistances of coils & $12.25 \mathrm{~m} \Omega, 17.03 \mathrm{~m} \Omega, 17.03 \mathrm{~m} \Omega, 17.03 \mathrm{~m} \Omega$ \\
\hline$R_{\mathrm{o} 2}, R_{\mathrm{o} 3}, R_{\mathrm{o} 4}$ & Load resistances & $4 \Omega, 8 \Omega, 10 \Omega$ \\
\hline$f_{1}$ & Operating frequency & $100 \mathrm{kHz}$ \\
\hline$f_{2}$ & Operating frequency & $5 \mathrm{kHz}$ \\
\hline$U_{\mathrm{o} 2}, U_{\mathrm{o} 3}, U_{\mathrm{o} 4}$ & Target voltage & $8 \mathrm{~V}, 10 \mathrm{~V}, 12 \mathrm{~V}$ \\
\hline$M_{23}, M_{24}, M_{34}$ & Mutual inductance & $0.9660 \mu \mathrm{H}$ \\
\hline
\end{tabular}




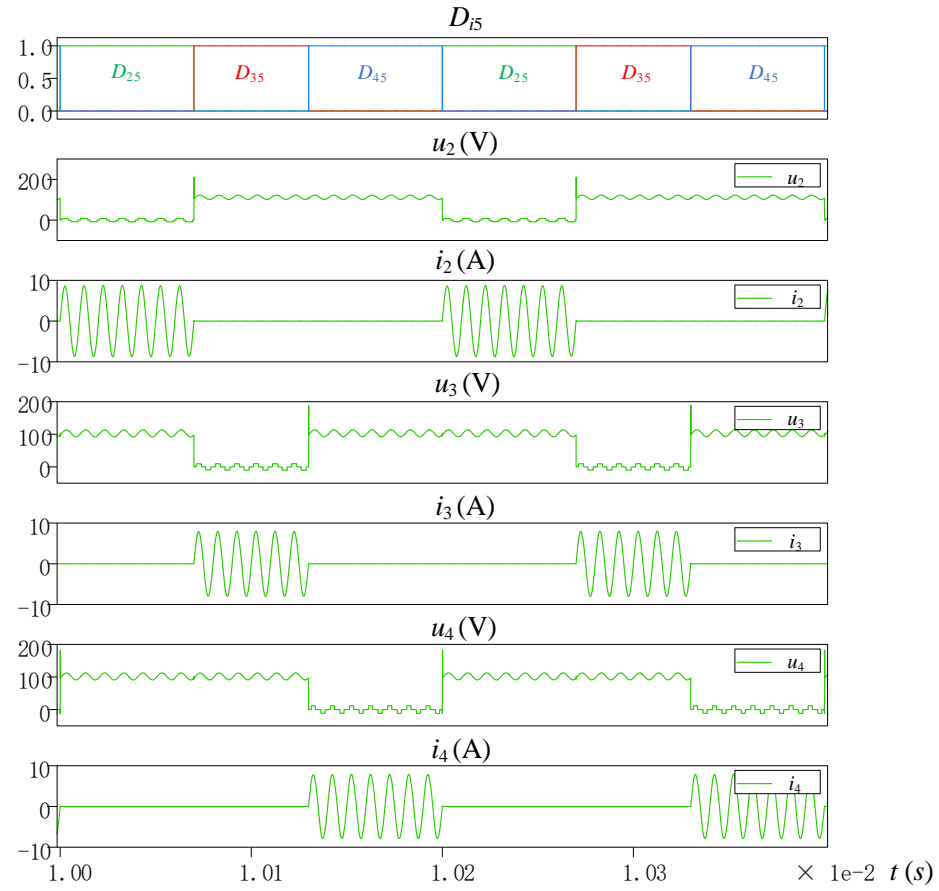

Figure 21. Simulation results of the time-sharing, three-receiver, wireless power transfer system with an output voltage control strategy.

\section{Conclusions}

This study developed a TS-MRWPT system to reduce the cross-coupling effect between receivers. First, a multiple-receiver system was simulated to illustrate that the cross-coupling between the receivers changed the magnetic field distribution. Second, the proposed time-sharing method in the TS-MRWPT was compared to the compensation method to verify that the time-sharing method had a high efficiency of about $93.2 \%$ for the simulation and about $82.4 \%$ for the experiment, which was close to the simulation efficiency of the compensation method of about $94.3 \%$. Moreover, further simulation comparisons between the time-sharing method and compensation method showed that the efficiency of the time-sharing method generally remained an $85.41 \%$, despite the variation of the distance between receivers, while the efficiency of the compensation method dropped rapidly from $88.33 \%$ to $77.66 \%$. The time-sharing method had a more flexible choice for the placement of the receiver coil. Finally, based on the proposed voltage control method, the TS-MRWPT system achieved output voltages of $7.29 \mathrm{~V}, 8.98 \mathrm{~V}$, and $11.03 \mathrm{~V}$, respectively, with target power voltages of $8 \mathrm{~V}, 10 \mathrm{~V}$, and $12 \mathrm{~V}$, respectively, and the efficiency was about $89.66 \%$ according to the simulation results.

Author Contributions: W.C. proposed the main idea, designed and performed the experiments, and wrote the manuscript. K.H. and J.X. double-checked and discussed the results and the whole manuscript. X.L., D.M., and H.T. contributed to the discussion of this study. All authors have approved the final version of this manuscript.

Funding: This research received no external funding.

Conflicts of Interest: The authors declare no conflict of interest.

\section{References}

1. Kuipers, J.; Bruning, H.; Bakker, S.; Rijnaarts, H. Near field resonant inductive coupling to power electronic devices dispersed in water. Sens. Actuators A Phys. 2012, 178, 217-222. [CrossRef]

2. Niu, W.; Gu, W.; Chu, J.; Shen, A. Frequency splitting of underwater wireless power transfer. In Proceedings of the IEEE International Workshop on Electromagnetics: Applications and Student Innovation Competition, Nanjing, China, 16-18 May 2016; pp. 1-3. 
3. Si, P.; Hu, A.P.; Malpas, S.; Budgett, D. A frequency control method for regulating wireless power to implantable devices. IEEE Trans. Biomed. Circuits Syst. 2008, 2, 22-29. [CrossRef]

4. Xue, R.F.; Cheng, K.W.; Je, M. High-Efficiency Wireless Power Transfer for Biomedical Implants by Optimal Resonant Load Transformation. IEEE Trans. Circuits Syst. I Regul. Pap. 2013, 60, 867-874. [CrossRef]

5. Meng, M.; Kiani, M. A Hybrid Inductive-Ultrasonic Link for Wireless Power Transmission to Millimeter-Sized Biomedical Implants. IEEE Trans. Circuits Syst. II Express Briefs 2016, 64, 1137-1141. [CrossRef]

6. Ramrakhyani, A.K.; Mirabbasi, S.; Mu, C. Design and optimization of resonance-based efficient wireless power delivery systems for biomedical implants. IEEE Trans. Biomed. Circuits Syst. 2011, 5, 48-63. [CrossRef] [PubMed]

7. Klontz, K.W.; Divan, D.M.; Novotny, D.W.; Lorenz, R.D. Contactless power delivery system for mining applications. IEEE Trans. Ind. Appl. 1995, 31, 27-35. [CrossRef]

8. Zhao, D.; Ding, E.J.; Xue, H. Multiple-Input Single-Output Wireless Power Transmission System for Coal Mine Application. Appl. Mech. Mater. 2014, 462-463, 900-904. [CrossRef]

9. Tampubolon, M.; Pamungkas, L.; Chiu, H.J.; Liu, Y.C.; Hsieh, Y.C. Dynamic Wireless Power Transfer for Logistic Robots. Energies 2018, 11, 527. [CrossRef]

10. Rajagopal, S.; Khan, F. Multiple Receiver Support for Magnetic Resonance Based Wireless Charging. In Proceedings of the 2011 IEEE International Conference on Communications Workshops (ICC), Kyoto, Japan, 5-9 June 2011; pp. 1-5.

11. Cannon, B.L.; Hoburg, J.F.; Stancil, D.D.; Goldstein, S.C. Magnetic Resonant Coupling As a Potential Means for Wireless Power Transfer to Multiple Small Receivers. IEEE Trans. Power Electron. 2009, 24, 1819-1825. [CrossRef]

12. Imura, T.; Hori, Y. Optimization using transmitting circuit of multiple receiving antennas for wireless power transfer via magnetic resonance coupling. In Proceedings of the 33rd International Telecommunications Energy Conference (INTELEC), Amsterdam, The Netherlands, 9-13 October 2011; pp. 1-4.

13. Lee, S.; Kim, S.; Seo, C. Design of multiple receiver for wireless power transfer using metamaterial. In Proceedings of the Asia-Pacific Microwave Conference Proceedings (APMC), Seoul, South Korea, 5-8 November 2013; pp. 1036-1038.

14. Cai, W.; Ma, D.; Tang, H.; Lai, X.; Liu, X.; Sun, L. Highly Efficient Target Power Control for Two-Receiver Wireless Power Transfer Systems. Energies 2018, 11, 2726. [CrossRef]

15. Sun, L.; Tang, H.; Zhong, S. Load-Independent Output Voltage Analysis of Multiple-Receiver Wireless Power Transfer System. IEEE Antennas Wirel. Propag. Lett. 2016, 15, 1238-1241. [CrossRef]

16. Fu, M.; Zhang, T.; Ma, C.; Zhu, X. Efficiency and Optimal Loads Analysis for Multiple-Receiver Wireless Power Transfer Systems. IEEE Trans. Microw. Theory Tech. 2015, 63, 801-812. [CrossRef]

17. Fu, M.; Yin, H.; Zhu, X.; Ma, C. Analysis and Tracking of Optimal Load in Wireless Power Transfer Systems. IEEE Trans. Power Electron. 2015, 30, 3952-3963. [CrossRef]

18. Berger, A.; Agostinelli, M.; Vesti, S.; Oliver, J.A.; Cobos, J.A.; Huemer, M. A Wireless Charging System Applying Phase-Shift and Amplitude Control to Maximize Efficiency and Extractable Power. IEEE Trans. Power Electron. 2015, 30, 6338-6348. [CrossRef]

19. Li, H.; Li, J.; Wang, K.; Chen, W. A Maximum Efficiency Point Tracking Control Scheme for Wireless Power Transfer Systems Using Magnetic Resonant Coupling. IEEE Trans. Power Electron. 2015, 30, 3998-4008. [CrossRef]

20. Li, Y.; Mai, R.; Liu, Y.; He, Z. Efficiency optimising strategy for dual-coupled transmitters based WPT systems. Electron. Lett. 2016, 52, 1877-1879. [CrossRef]

21. Liu, X.; Wang, T.; Yang, X.; Jin, N.; Tang, H. Analysis and Design of a Wireless Power Transfer System with Dual Active Bridges. Energies 2017, 10, 1588. [CrossRef]

22. Ahn, D.; Hong, S. Effect of Coupling Between Multiple Transmitters or Multiple Receivers on Wireless Power Transfer. IEEE Trans. Ind. Electron. 2013, 60, 2602-2613. [CrossRef]

23. Kim, J.; Son, H.; Kim, D.; Park, Y. Impedance matching considering cross coupling for wireless power transfer to multiple receivers. In Proceedings of the 2013 IEEE Wireless Power Transfer (WPT), Perugia, Italy, 15-16 May 2013; pp. 226-229.

24. Cui, D.; Imura, T.; Hori, Y. Cross coupling cancellation for all frequencies in multiple-receiver wireless power transfer systems. In Proceedings of the International Symposium on Antennas and Propagation (ISAP), Okinawa, Japan, 24-28 October 2016; pp. 48-49. 
25. Fu, M.; Zhang, T.; Zhu, X.; Luk, P.C.; Ma, C. Compensation of Cross Coupling in Multiple-Receiver Wireless Power Transfer Systems. IEEE Trans. Ind. Inf. 2016, 12, 474-482. [CrossRef]

26. Jiang, C.; Chau, K.T.; Liu, C.; Han, W. Wireless DC Motor Drives with Selectability and Controllability. Energies 2017, 10, 49. [CrossRef]

27. Kim, Y.; Ha, D.; Chappell, W.J.; Irazoqui, P.P. Selective Wireless Power Transfer for Smart Power Distribution in a Miniature-Sized Multiple-Receiver System. IEEE Trans. Ind. Electron. 2016, 63, 1853-1862. [CrossRef]

28. Yanagawa, S.; Shimizu, R.; Hamada, M.; Kuroda, T. Wireless Power Transfer System for 3-D stacked Multiple Receivers Switching between Single and Dual Frequency Modes. In Proceedings of the 61st International Midwest Symposium on Circuits and Systems (MWSCAS), Windsor, ON, Canada, 5-8 August 2018; pp. 1046-1049.

29. Yin, H.; Fu, M.; Zhao, C.; Ma, C. A decentralized charging control of a multiple-receiver wireless power transfer system using ultracapacitor semi-active topology. In Proceedings of the 25th International Symposium on Industrial Electronics (ISIE), Santa Clara, CA, USA, 8-10 June 2016; pp. 262-267.

30. Jiang, C.; Chau, K.T.; Ching, T.W.; Liu, C.; Han, W. Time-Division Multiplexing Wireless Power Transfer for Separately Excited DC Motor Drives. IEEE Trans. Magn. 2017, 53, 1-5. [CrossRef]

31. Juliet, V.; Padmanaban, S.; Mihet-Popa, L. Frequency Splitting Elimination and Cross-Coupling Rejection of Wireless Power Transfer to Multiple Dynamic Receivers. Appl. Sci. 2018, 8, 179. [CrossRef]

32. Deng, J.; Deng, J.; Li, W.; Li, S.; Mi, C. Magnetic integration of LCC compensated resonant converter for inductive power transfer applications. In Proceedings of the Energy Conversion Congress and Exposition (ECCE), Pittsburgh, PA, USA, 14-18 September 2014; pp. 660-667.

(C) 2020 by the authors. Licensee MDPI, Basel, Switzerland. This article is an open access article distributed under the terms and conditions of the Creative Commons Attribution (CC BY) license (http://creativecommons.org/licenses/by/4.0/). 